\title{
Bifurcation and segregation in quadratic two-populations Mean Field Games systems
}

\author{
Marco Cirant and Gianmaria Verzini
}

September 28, 2018

\begin{abstract}
We search for non-constant normalized solutions to the semilinear elliptic system

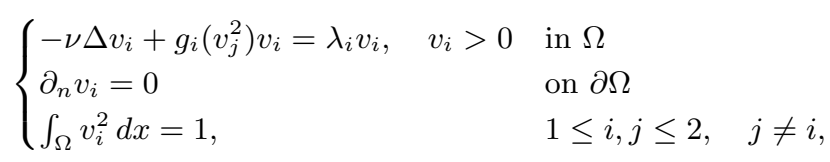

where $\nu>0, \Omega \subset \mathbb{R}^{N}$ is smooth and bounded, the functions $g_{i}$ are positive and increasing, and both the functions $v_{i}$ and the parameters $\lambda_{i}$ are unknown. This system is obtained, via the Hopf-Cole transformation, from a two-populations ergodic Mean Field Games system, which describes Nash equilibria in differential games with identical players. In these models, each population consists of a very large number of indistinguishable rational agents, aiming at minimizing some long-time average criterion.

Firstly, we discuss existence of nontrivial solutions, using variational methods when $g_{i}(s)=s$, and bifurcation ones in the general case; secondly, for selected families of nontrivial
\end{abstract} solutions, we address the appearing of segregation in the vanishing viscosity limit, i.e.

$$
\int_{\Omega} v_{1} v_{2} \rightarrow 0 \quad \text { as } \nu \rightarrow 0
$$

AMS-Subject Classification. 35J47, 49N70, 35B25, 35B32

Keywords. Singularly perturbed problems, normalized solutions to semilinear elliptic systems, multi-population differential games

\section{Introduction}

We consider the following semilinear elliptic system

$$
\begin{cases}-\nu \Delta v_{1}+g_{1}\left(v_{2}^{2}\right) v_{1}=\lambda_{1} v_{1} & \\ -\nu \Delta v_{2}+g_{2}\left(v_{1}^{2}\right) v_{2}=\lambda_{2} v_{2} & \text { in } \Omega \\ \int_{\Omega} v_{1}^{2} d x=\int_{\Omega} v_{2}^{2} d x=1, \quad v_{1}, v_{2}>0 & \\ \partial_{n} v_{1}=\partial_{n} v_{2}=0 & \text { on } \partial \Omega .\end{cases}
$$

Here $\Omega \subset \mathbb{R}^{N}$ is a smooth bounded domain, normalized in such a way that

$$
|\Omega|=1,
$$


$\nu>0$, and both the functions $v_{i}$ and the parameters $\lambda_{i}$ are unknown. The interaction functions $g_{i} \in C^{2}([0, \infty))$ satisfy

$$
\begin{aligned}
& \text { - } C_{g}^{-1} s \leq g_{i}(s) \leq C_{g} s \quad \forall s \geq 0, \\
& \text { - } g_{i} \text { is strictly increasing, } \quad g_{i}^{\prime}(1)>0 \text {, }
\end{aligned}
$$

for some $C_{g}>0(i=1,2)$.

The elliptic system (1) arises in the context of Mean Field Games (briefly MFG) theory. MFG is a branch of Dynamic Games which has been proposed independently by Lasry, Lions [21, 22, 23] and Caines, Huang, Malhamé [19, 18] in the engineering community, with the aim of modeling and analyzing decision processes involving a very large number of indistinguishable rational agents. Here, we focus on MFG with two competing populations, where every individual of the $i$-th population $(i=1,2)$ is represented by a typical agent, and whose state is driven by the controlled stochastic differential equation

$$
d X_{s}^{i}=-a_{s}^{i} d s+\sqrt{2 \tilde{\nu}} d B_{s}^{i},
$$

where $B_{s}^{i}$ are independent Brownian motions. The agent chooses her own velocity $a_{s}^{i}$ in order to minimize a cost of long-time-average form

$$
\mathcal{J}^{i}\left(X_{0}^{i}, a^{1}, a^{2}\right)=\liminf _{T \rightarrow \infty} \frac{1}{T} \int_{0}^{T} \mathbb{E}\left[\frac{\left|a_{s}^{i}\right|^{2}}{2}+g_{i}\left(\left(\hat{m}_{j}\right)_{s}\right)\right] d s,
$$

where $\hat{m}_{j}$ denotes the empirical density of the players belonging to the other population (i.e. $j=3-i$ ). It has been shown (see in particular [16]) that equilibria of the game (in the sense of Nash) are captured by the following system of non-linear elliptic equations

$$
\begin{cases}-\tilde{\nu} \Delta u_{i}(x)+\frac{1}{2}\left|\nabla u_{i}(x)\right|^{2}+\lambda_{i}=g_{i}\left(m_{j}(x)\right) & \\ -\tilde{\nu} \Delta m_{i}(x)-\operatorname{div}\left(\nabla u_{i}(x) m_{i}(x)\right)=0 & \text { in } \Omega \\ \int_{\Omega} m_{i} d x=1, m_{i}>0, & i=1,2 .\end{cases}
$$

The unknowns $u_{i}, \lambda_{i}$ provide the value functions of typical players and the average costs respectively. On the other hand, the unknowns $m_{i}$ represent the stationary distributions of players of the $i$-th population implementing the optimal strategy, that is the long time behavior of agents playing in an optimal way. We suppose that the state $X_{s}^{i}$ is subject to reflection at $\partial \Omega$; this motivates the Neumann boundary conditions.

Note that the individual cost $\mathcal{J}^{i}$ is increasing with respect to $\hat{m}_{j}$, as we are supposing that $g_{i}$ is increasing. In other words, every agent is lead to avoid regions of $\Omega$ where an high concentration of competitors is present. For this reason, our MFG model is expected to show phenomena of segregation between the two populations. In particular, segregation should arise distinctly in the vanishing viscosity regime, namely when the Brownian noise (whose intensity is controlled by $\tilde{\nu}$ ) becomes negligible with respect to interactions. We will explore this aspect in terms of qualitative properties of the two distributions $m_{1}, m_{2}$.

Another key feature of this model is the quadratic dependence of the cost $\mathcal{J}^{i}$ with respect to the velocity $\alpha_{i}$. It has been pointed out (see 21, 24]) that the so-called Hopf-Cole transformation partially decouples the equations in (3), reducing the number of the unknowns. Precisely, if we let

$$
v_{i}^{2}:=m_{i}=e^{-u_{i} / \tilde{\nu}} \quad \text { and } \quad \nu=2 \tilde{\nu}^{2}
$$

then (3) becomes (11). We will therefore consider (1) and transpose the obtained results to the original system (3). 
Before proceeding with the analysis of the reduced system (1), a few bibliographical remarks are in order. First of all, while the single population case has received a considerable attention, few papers deal with mathematical aspects of the multi-population setting. We mention that a preliminary study of (1)-(3) has been made in [10, while a non-stationary version of (3) is considered in [20]. The latter work provides also a motivation for (3) based on pedestrian crowd models. Our MFG system can be also seen as a simplified version of the population models presented in [1].

Since $|\Omega|=1$,

$$
v_{1} \equiv v_{2} \equiv 1, \quad \lambda_{1}=g_{1}(1), \quad \lambda_{2}=g_{2}(1)
$$

is a solution of (11) for every value of $\nu$. We will refer to it (or, with some abuse, to the pair $\left.\left(v_{1}, v_{2}\right) \equiv(1,1)\right)$ as the trivial (or constant) solution. The aim of our investigation is twofold: firstly, to show the existence of families, indexed by $\nu$, of nontrivial Nash equilibria for (1); secondly, to analyze possible segregation phenomena for such families, as $\nu \rightarrow 0$.

Definition 1.1. The pair $\left(v_{1}, v_{2}\right)$ is a Nash equilibrium for (1) if each $v_{i}$ achieves

$$
\lambda_{i}:=\inf \left\{\int_{\Omega}\left[\nu|\nabla w|^{2}+g_{i}\left(v_{j}^{2}\right) w^{2}\right] d x: w \in H^{1}(\Omega), \int_{\Omega} w^{2} d x=1\right\} .
$$

It is easy to show (see Lemma 2.1 ahead) that a pair $\left(v_{1}, v_{2}\right)$ is a Nash equilibrium if and only if (up to a change of sign of its components) it solves (1) with multipliers $\left(\lambda_{1}, \lambda_{2}\right)$.

Definition 1.2. We say that a set of solutions

$$
\Sigma \subset\left\{\left(\nu, v_{1}, v_{2}\right) \in \mathbb{R} \times C^{2, \alpha}(\bar{\Omega}) \times C^{2, \alpha}(\bar{\Omega}):\left(\nu, v_{1}, v_{2}\right) \text { satisfies (1) for some }\left(\lambda_{1}, \lambda_{2}\right)\right\}
$$

segregates if it contains sequences $\left\{\left(\nu_{n}, v_{1, n}, v_{2, n}\right)\right\}_{n}$ with $\nu_{n} \rightarrow 0$, and for every such sequence it holds

$$
\int_{\Omega} v_{1, n} v_{2, n} \rightarrow 0 \quad \text { as } n \rightarrow \infty
$$

One important feature of system (1) is that its unknowns are both the functions $v_{i}$, which are required to be normalized (in the $L^{2}$ sense), and the parameters $\lambda_{i}$. Despite the large literature devoted to existence results for semilinear elliptic systems, only few papers deal with normalized solutions, mainly when searching for solitary waves associated to nonlinear Schrödinger systems [26, 27, 28, 4, 3]. Note that all these papers are based on variational methods, since the systems they consider are of gradient type. This is not the case for (1), except when the interactions $g_{i}$ are linear functions.

On the other hand, segregation issues have received much attention in the last decade, and by now a large amount of literature is dedicated to this subject, see e.g. [11, 9, 12, 8, 17, 33, 25, 15. 6, 29, 32, the recent survey [31, and references therein. Mainly two types of competitions have been widely investigated, namely the Lotka-Volterra type (e.g. $\left.g_{i}(s)=a_{i} \sqrt{s}\right)$, and again the variational one. Furthermore in these papers segregation (as defined in Definition 1.2) is a first easy step, while all the effort is done to show that the convergence of $v_{1} v_{2}$ to 0 is very much stronger than merely $L^{1}$. Conversely, in our situation, even the $L^{1}$ convergence is not clear at all, mainly due to the unknown behavior of the parameters $\lambda_{i}$. For instance, the set of trivial solutions does not segregate at all. Actually, this is one of the main difficulties we have to face.

Motivated by the above discussion, we first treat the variational case

$$
g_{i}(s)=\gamma_{i} s, \quad \text { for some } \gamma_{i}>0
$$


In such a case, as we mentioned, (1) has a gradient structure, at least in dimension $N \leq 3$ : Nash equilibria can be obtained as critical points of the functional

$$
I_{\nu}\left(v_{1}, v_{2}\right)=\int_{\Omega}\left[\frac{1}{\gamma_{1}}\left|\nabla v_{1}\right|^{2}+\frac{1}{\gamma_{2}}\left|\nabla v_{2}\right|^{2}+\frac{1}{\nu} v_{1}^{2} v_{2}^{2}\right] d x
$$

constrained to the manifold

$$
M=\left\{\left(v_{1}, v_{2}\right) \in H^{1}(\Omega) \times H^{1}(\Omega): \int_{\Omega} v_{1}^{2} d x=\int_{\Omega} v_{1}^{2} d x=1\right\} .
$$

As a consequence, existence of solutions can be obtained by direct minimization of $\left.I_{\nu}\right|_{M}$. Regarding the asymptotic behaviour of such minimizers, using techniques contained in [26] we can show $\Gamma$-convergence to the following limiting problem:

$$
\min \left\{\int_{\Omega}\left[\frac{1}{\gamma_{1}}\left|\nabla v_{1}\right|^{2}+\frac{1}{\gamma_{2}}\left|\nabla v_{2}\right|^{2}\right] d x:\left(v_{1}, v_{2}\right) \in M, v_{1} \cdot v_{2} \equiv 0,\right\} .
$$

It can be proved that such minimum is achieved, and, among other properties, that any minimizer $\left(V_{1}, V_{2}\right)$ is such that $V_{1} \sqrt{\gamma_{2}}-V_{2} \sqrt{\gamma_{1}} \in C^{2, \alpha}(\bar{\Omega})$, for every $0<\alpha<1$ (see Proposition $3.3 \mathrm{ahead}$ ). As a matter of fact, we can prove the following.

Theorem 1.3 (Variational case). Let $N \leq 3, g_{i}(s)=\gamma_{i} s, \gamma_{i}>0$, and let $\mu_{1}>0$ denote the first positive Neumann eigenvalue of $-\Delta$ in $\Omega$. Then, for every

$$
0<\nu \leq \frac{\gamma_{1} \gamma_{2}}{\mu_{1}\left(\gamma_{1}+\gamma_{2}\right)}
$$

the minimum of $\left.I_{\nu}\right|_{M}$ is achieved by a pair $\left(v_{1, \nu}, v_{2, \nu}\right)$, which is a nontrivial Nash equilibrium for (11).

Moreover, any family of minimizers exhibits segregation: up to subsequences,

$$
v_{i, \nu} \rightarrow V_{i} \text { in } H^{1}(\Omega) \cap C^{\alpha}(\bar{\Omega}) \quad \text { as } \nu \rightarrow 0
$$

for every $\alpha<1$, where $\left(V_{1}, V_{2}\right)$ achieves (4).

Turning to the general case, since (10) has no variational structure, one is lead to search for solutions using topological methods. In particular, it is natural to use bifurcation theory to find nontrivial solutions $\left(\nu, v_{1}, v_{2}\right)$ branching off from the trivial ones

$$
\mathcal{T}=\{(\nu, 1,1): \nu>0\} \subset \mathbb{R} \times C^{2, \alpha}(\bar{\Omega}) \times C^{2, \alpha}(\bar{\Omega}) .
$$

We denote by $\mathcal{S}$ the closure of the set of nontrivial solutions of (11), so that a bifurcation point is a point of $\mathcal{S} \cap \mathcal{T}$. The classical bifurcation theory by Rabinowitz [30, 14] can be applied to our setting to obtain the following.

Theorem 1.4. Let $\mu^{*}>0$ denote a positive Neumann eigenvalue of $-\Delta$ in $\Omega$, and

$$
\nu^{*}=\frac{2 \sqrt{g_{1}^{\prime}(1) g_{2}^{\prime}(1)}}{\mu^{*}} .
$$

- If $\mu^{*}$ has odd multiplicity then there exists a continuum $\mathcal{C}^{*} \subset \mathcal{S}$ such that $\left(\nu^{*}, 1,1\right) \in \mathcal{C}^{*}$ and 
- either $\left(\nu^{* *}, 1,1\right) \in \mathcal{C}^{*}$, where $\nu^{* *}=2 \sqrt{g_{1}^{\prime}(1) g_{2}^{\prime}(1)} / \mu^{* *}$ and $\mu^{* *} \neq \mu^{*}$ is another positive Neumann eigenvalue,

- or $\mathcal{C}^{*}$ is unbounded; furthermore, in dimension $N \leq 3, \mathcal{C} \cap\left\{\left(\nu, v_{1}, v_{2}\right): \nu \geq \bar{\nu}\right\}$ is bounded for every $\bar{\nu}>0$, and $\mathcal{C}$ contains a sequence $\left(\nu_{n}, v_{1, n}, v_{2, n}\right)$ such that, as $n \rightarrow+\infty$,

$$
\nu_{n} \rightarrow 0, \quad\left\|\left(v_{1, n}, v_{2, n}\right)\right\|_{C^{2, \alpha}} \rightarrow+\infty .
$$

- If $\mu^{*}$ is simple (with eigenfunction $\psi^{*}$ ) then the set of non-trivial solutions is, near $\left(\nu^{*}, 1,1\right)$, a unique smooth curve with parametric representation

$$
\nu=\nu(\varepsilon), \quad v=(1,1)+\varepsilon v^{*}+o(\varepsilon)
$$

where $\nu(0)=\nu^{*}$ and $v^{*}=\left(-\psi^{*} \sqrt{g_{1}^{\prime}(1)}, \psi^{*} \sqrt{g_{2}^{\prime}(1)}\right)$.

Remark 1.5. Sharper asymptotic expansions are provided in Remark 4.6 ahead, in case both $g_{i}$ are more regular.

Remark 1.6. To compare this theorem with the classical results by Rabinowitz, we recall that here the natural bifurcation parameter is $1 / \nu$, rather than $\nu$ itself. In particular, in case infinitely many eigenvalues $\mu_{n}$ are odd, we have infinitely many bifurcation points $\nu_{n} \rightarrow 0$. As a consequence it is easy to construct families of nontrivial solutions, jumping from branch to branch, that not only do not exhibit segregation, but even tend to the trivial solution as $\nu \rightarrow 0$.

The previous remark shows that one can not expect segregation for a generic family of nontrivial solutions. It is then natural to ask whether segregation occurs for the bifurcation branches above described, at least for the unbounded ones. According to Theorem 1.4, in order to find unbounded branches of nontrivial solutions we first have to find odd eigenvalues of $-\Delta$ in $\Omega$, and then to exclude that the corresponding branch goes back to the set of trivial solutions. Usually, in the bifurcation framework, both conditions can be satisfied when working with the first eigenvalue of the linearized problem: indeed, on one hand such eigenvalue is simple; on the other hand, it is usually possible to carry over to the full branch the nodal characterization of the corresponding eigenfunction. Notice that this is not our case, since the first Neumann eigenvalue is 0 and it does not provide a bifurcation point, while the first positive eigenvalue $\mu_{1}$ is actually the second one. Another way to exploit these ideas is to work in dimension $N=1$.

Theorem 1.7. Let $N=1$. For any $k \in \mathbb{N}, k \geq 1$ there exists a continuum $\mathcal{C}_{k}$ of solutions, such that:

- if $\left(\nu, v_{1}, v_{2}\right) \in \mathcal{C}_{k}$ then both $v_{i}$ have exactly $k-1$ critical points;

- $\overline{\mathcal{C}_{k}} \cap \mathcal{T}=\left\{\left(\frac{2 \sqrt{g_{1}^{\prime}(1) g_{2}^{\prime}(1)}}{\pi^{2} k^{2}}, 1,1\right)\right\}$;

- $h \neq k$ implies $\mathcal{C}_{h} \cap \mathcal{C}_{k}=\emptyset$;

- each $\mathcal{C}_{k}$ contains sequences with $\nu \rightarrow 0$ and $\left\|\left(v_{1}, v_{2}\right)\right\|_{C^{2, \alpha}} \rightarrow+\infty$;

- each $\mathcal{C}_{k}$ segregates.

Once segregation is obtained, we have that the segregating branches converge, up to subsequences, to some limiting profiles. As a consequence, some natural questions arise, about the type of convergence as well as about the properties of the limiting profiles. We can give the full picture in the case of the first branch. 
Theorem 1.8. Let $N=1$ and $\mathcal{C}_{1}$ as in Theorem 1.7. Then, any sequence $\left\{\left(\nu_{n}, v_{n}, \lambda_{n}\right)\right\}_{n} \subset \mathcal{C}_{1}$ such that $\nu_{n} \rightarrow 0$ is uniformly bounded in Lipschitz norm, and it holds

$$
v_{i, n} \rightarrow V_{i} \text { in } H^{1}(\Omega) \cap C^{\alpha}(\bar{\Omega}) \quad \text { as } \nu_{n} \rightarrow 0,
$$

for every $\alpha<1$, where $\left(V_{1}, V_{2}\right)$ is the minimizer (unique up to reflections) achieving (4) with $\gamma_{i}=g_{i}^{\prime}(0) \geq C_{g}^{-1}$.

Remark 1.9. We expect that most of the results of Theorems 1.7 and 1.8 can be extended to higher dimension, in the radial setting.

It is easy to see that the convergence above is optimal: indeed, in case of Lipschitz convergence, both $V_{i}$ would be $C^{1}$, a contradiction with their explicit expression provided in Proposition 3.3. Up to our knowledge, this is the first paper obtaining optimal bounds for competitions which are not of power-type, even though only in dimension $N=1$ (or in the radial case). The only other paper dealing with generic competitions is [33], where uniform bounds in the planar case $N=2$, not necessarily radial, are obtained.

Let us also point out that along the first branch the problem -which is not variational-inherits a variational principle in the limit. This is a remarkable fact, since it shows a deep connection between the variational problem (4) and the nonvariational system (1). This phenomenon was already observed, in a different situation, in [13].

Of course, all the results we obtained for system (1) can be restated for the original MFG system (3), recalling that

$$
m_{i}=v_{i}^{2}, \quad u_{i}=-2 \tilde{\nu} \ln v_{i} .
$$

Finally, let us also mention that the true multidimensional case $N \geq 2$, as well as the case of 3 or more populations, are of interest: they will be the object of future studies.

The present paper is structured as follows: in Section 2 we list a few preliminary results; Section 3 is devoted to the analysis of the variational case, and to the proof of Theorem 1.3, while Section 4 contains the bifurcation arguments and the proof of Theorem 1.4 the Sturm-type characterization of the nontrivial solutions in dimension $N=1$ is developed in Section [5 and the proof of Theorem 1.7 is completed in Section 6 , by showing segregation; finally, the proof of Theorem 1.8 is contained in Section 7 .

Notation. Throughout the paper, $i$ denotes an index between 1 and 2 , and $j=3-i$. With a little abuse of terminology, we say that $\left(v_{1}, v_{2}\right)$ solves (11) (or even that $\left(\nu, v_{1}, v_{2}\right)$ does) if there exist $\lambda_{1}, \lambda_{2}$ such that $\left(v_{1}, v_{2}, \lambda_{1}, \lambda_{2}\right)$ satisfies (11) (for some prescribed $\nu$ ).

We will denote by $\left(\mu_{k}\right)_{k \geq 0}$ the non decreasing sequence of the eigenvalues of $-\Delta$ with homogeneous Neumann boundary conditions, namely $\mu_{k}$ is such that

$$
\begin{cases}-\Delta \psi_{k}=\mu_{k} \psi_{k} & \text { in } \Omega \\ \partial_{n} \psi_{k}=0 & \text { on } \partial \Omega,\end{cases}
$$

for some eigenvector $\psi_{k} \in C^{2, \alpha}(\bar{\Omega})$, which constitute an orthonormal basis of $L^{2}(\Omega)$. The first eigenvalue $\mu_{0}=0$ is simple and its corresponding eigenfunction is $\psi \equiv 1$.

Given a function $u, u^{ \pm}(x)=\max ( \pm u(x), 0)$ denote its positive and negative parts. Finally, $C, C_{1}, C_{2}, \ldots$ denote (positive) constants we need not to specify.

\section{Preliminaries}

In this section we collect some preliminary results and some estimates of frequent use. 
Lemma 2.1. The pair $\left(v_{1}, v_{2}\right)$ is a Nash equilibrium if and only if, up to a change of sign of each component, it is a (classical) solution of (1).

Proof. Considering $v_{j}$ as fixed, we have that $v_{i}$ is an $L^{2}$-normalized eigenfunction of the Neumann realization of the operator

$$
H^{1}(\Omega) \ni w \mapsto-\nu \Delta w+g_{i}\left(v_{j}^{2}\right) w,
$$

and that $\lambda_{i}$ is the corresponding eigenvalue. But then $v_{i}$ is strictly positive (up to a change of sign) if and only if it is the first eigenfunction, i.e. it achieves the infimum in Definition 1.1 . In particular, the proof of the strict positivity in $\bar{\Omega}$ is a routine application of the Maximum Principle and Hopf's Lemma.

Lemma 2.2. Let $\left(v_{1}, v_{2}\right)$ solve (11). Then either it is trivial, or

$$
\min _{\bar{\Omega}} g_{i}\left(v_{j}^{2}\right)<\lambda_{i}<\max _{\bar{\Omega}} g_{i}\left(v_{j}^{2}\right), \quad i=1,2 .
$$

Proof. Integrating the equation for $v_{i}$ we can write

$$
\int_{\Omega}\left[\lambda_{i}-g_{i}\left(v_{j}^{2}\right)\right] v_{i} d x=\nu \int_{\partial \Omega} \partial_{n} v_{i} d \sigma=0,
$$

and since $v_{i}$ is positive, we deduce that either $\lambda_{i}-g_{i}\left(v_{j}^{2}\right) \equiv 0$, i.e. $v_{j}$ is constant, or $\min _{\bar{\Omega}} g_{i}\left(v_{j}^{2}\right)<$ $\lambda_{i}<\max _{\bar{\Omega}} g_{i}\left(v_{j}^{2}\right)$.

Now, if both $v_{i}$ and $v_{j}$ are not constant, then the second alternative follows. Let $v_{i}$ be constant: then its equation implies $g_{i}\left(v_{j}^{2}\right) \equiv \lambda_{i}$, so that also $v_{j}$ is constant. Finally, both such constants must be 1 by the $L^{2}$ - constraint (recall that $|\Omega|=1$ ).

Remark 2.3. The above lemma shows that, for Nash equilibria, having a constant component implies being the trivial solution (in this sense, the terminology "constant solution" is not ambiguous). In fact, if unique continuation for (10) holds, then any solution such that one component is constant in a (non empty) open $\Omega_{0} \subset \Omega$ must be the trivial one. This is always true, in particular, in dimension $N=1$ (see Section 5 ).

Lemma 2.4. Let $\left(v_{1}, v_{2}\right)$ solve (1). The following identities hold, for every $i$ :

$$
\begin{aligned}
& \nu \int_{\Omega}\left|\nabla v_{i}\right|^{2}+\int_{\Omega} g_{i}\left(v_{j}^{2}\right) v_{i}^{2}=\lambda_{i} \\
& \nu \int_{\Omega}\left|\frac{\nabla v_{i}}{v_{i}}\right|^{2}+\lambda_{i}=\int_{\Omega} g_{i}\left(v_{j}^{2}\right) .
\end{aligned}
$$

In particular, the multipliers $\lambda_{i}$ satisfy

$$
C_{g}^{-1} \int_{\Omega} v_{1}^{2} v_{2}^{2} \leq \lambda_{i} \leq C_{g}
$$

Proof. To obtain the two identities it suffices to use integration by parts after multiplying the equation for $v_{i}$ by $v_{i}$ and $1 / v_{i}$, respectively. Since $\int_{\Omega} v_{i}^{2}=1$ and $C_{g}^{-1} s \leq g_{i}(s) \leq C_{g} s$, (5) follows.

Corollary 2.5. A sufficient condition for $\left\{\left(\nu_{n}, v_{1, n}, v_{2, n}\right)\right\}_{n}$ to segregate is that, for the corresponding multipliers,

$$
\text { either } \lambda_{1, n} \rightarrow 0, \quad \text { or } \lambda_{2, n} \rightarrow 0,
$$

as $n \rightarrow \infty$. 


\section{The variational case}

This section is devoted to the proof of Theorem 1.3. Such proof relies on ideas contained in [26, even though in that paper a different problem is considered (Dirichlet conditions, symmetric interaction, auto-catalytic reaction terms). For this reason we describe the main ideas here, and refer the reader to 26 for more details.

In the following we assume that $N \leq 3$ and

$$
g_{i}(s)=\gamma_{i} s, \quad \gamma_{i}>0 .
$$

As we already noticed, the corresponding system has a gradient structure. For easier notation we make a change of variable, setting

$$
\beta=\frac{1}{\nu}, \quad \tilde{v}_{1}=\sqrt{\gamma_{2}} v_{1}, \quad \tilde{v}_{2}=\sqrt{\gamma_{1}} v_{2} .
$$

With this notation system (1) becomes

$$
\begin{cases}-\Delta \tilde{v}_{1}+\beta \tilde{v}_{2}^{2} \tilde{v}_{1}=\lambda_{1} \tilde{v}_{1} & \\ -\Delta \tilde{v}_{2}+\beta \tilde{v}_{1}^{2} \tilde{v}_{2}=\lambda_{2} \tilde{v}_{2} & \text { in } \Omega \\ \int_{\Omega} \tilde{v}_{1}^{2}=\gamma_{2}, \int_{\Omega} \tilde{v}_{2}^{2}=\gamma_{1}, \quad \tilde{v}_{1}, \tilde{v}_{2}>0 & \\ \partial_{n} \tilde{v}_{1}=\partial_{n} \tilde{v}_{2}=0 & \text { on } \partial \Omega\end{cases}
$$

(of course, the multipliers $\lambda_{i}$ here are suitable multiple of those of the original system). Also for (17) positive solutions are Nash equilibria, among which the trivial one is the pair $\left(\sqrt{\gamma_{2}}, \sqrt{\gamma_{1}}\right)$. Solutions to (7D) are critical points of the functional

$$
J_{\beta}\left(\tilde{v}_{1}, \tilde{v}_{2}\right)=\int_{\Omega}\left[\left|\nabla \tilde{v}_{1}\right|^{2}+\left|\nabla \tilde{v}_{2}\right|^{2}+\beta \tilde{v}_{1}^{2} \tilde{v}_{2}^{2}\right]
$$

constrained to the manifold

$$
\tilde{M}=\left\{\left(\tilde{v}_{1}, \tilde{v}_{2}\right) \in H^{1}(\Omega) \times H^{1}(\Omega): \int_{\Omega} \tilde{v}_{1}^{2}=\gamma_{2}, \int_{\Omega} \tilde{v}_{2}^{2}=\gamma_{1}\right\}
$$

(recall that, since $N \leq 3$, the exponent $p=4$ is Sobolev subcritical and thus $J_{\beta}$ is of class $C^{1}$ ).

Lemma 3.1. For every $\beta>0$ the value

$$
c_{\beta}:=\inf _{\tilde{M}} J_{\beta} \quad \text { is achieved by } \quad\left(\tilde{v}_{1, \beta}, \tilde{v}_{2, \beta}\right) \in \tilde{M},
$$

which is a Nash equilibrium for (7). Furthermore, if

$$
\beta \geq \frac{\gamma_{1}+\gamma_{2}}{\gamma_{1} \gamma_{2}} \mu_{1}
$$

(the first positive Neumann eigenvalue of $-\Delta$ in $\Omega$ ) then $\left(\tilde{v}_{1, \beta}, \tilde{v}_{2, \beta}\right)$ is nontrivial.

Proof. Since $J_{\beta}$ is weakly l.s.c. in $H^{1}$, and $\tilde{M}$ is weakly closed, the minima $\left(\tilde{v}_{1, \beta}, \tilde{v}_{2, \beta}\right)$ exist by the direct method. Moreover, since

$$
\int_{\Omega}\left[\left|\nabla \tilde{v}_{i}\right|^{2}+\beta \tilde{v}_{j}^{2} \tilde{v}_{i}^{2}\right]=J_{\beta}\left(\tilde{v}_{1}, \tilde{v}_{2}\right)-\int_{\Omega}\left|\nabla \tilde{v}_{j}\right|^{2},
$$


we have that such minima correspond to Nash equilibria for the original problem (the converse, of course, is false). We are left to prove that, for $\beta$ large, $\left(\tilde{v}_{1, \beta}, \tilde{v}_{2, \beta}\right) \neq\left(\sqrt{\gamma_{2}}, \sqrt{\gamma_{1}}\right)$. To do that, we will choose a suitable competitor in the definition of $c_{\beta}$ : let $\psi_{1}$ be an eigenfunction associated to $\mu_{1}$. Then $\psi_{1}$ changes sign (indeed it is orthogonal to the eigenfunction $\psi_{0}=1$, associated to $\left.\mu_{0}=0\right)$ and we can find non-zero constants $a_{ \pm}$such that $\left(a_{+} \psi^{+}, a_{-} \psi^{-}\right) \in \tilde{M}$. Then

$$
c_{\beta}<J_{\beta}\left(a_{+} \psi^{+}, a_{-} \psi^{-}\right)=\left(\gamma_{1}+\gamma_{2}\right) \mu_{1}
$$

(equality can not hold since $\left(a_{+} \psi^{+}, a_{-} \psi^{-}\right)$can not solve (7D) while

$$
J_{\beta}\left(\sqrt{\gamma_{2}}, \sqrt{\gamma_{1}}\right)=\gamma_{1} \gamma_{2} \beta
$$

Once we have solved the problem for $\beta>0$ fixed, we are ready to show $\Gamma$-convergence as $\beta \rightarrow+\infty$. Let

$$
J_{\infty}\left(\tilde{v}_{1}, \tilde{v}_{2}\right):=\left\{\begin{array}{ll}
\int_{\Omega}\left[\left|\nabla \tilde{v}_{1}\right|^{2}+\left|\nabla \tilde{v}_{2}\right|^{2}\right] & \text { when } \int_{\Omega} \tilde{v}_{1}^{2} \tilde{v}_{2}^{2}=0 \\
+\infty & \text { otherwise }
\end{array} \quad \text { and } \quad c_{\infty}:=\inf _{\tilde{M}} J_{\infty} .\right.
$$

Lemma 3.2. As $\beta \rightarrow+\infty$,

$$
c_{\beta} \rightarrow c_{\infty} \quad \text { and (up to subs.) } \quad \tilde{v}_{i, \beta} \rightarrow \tilde{V}_{i} \text { in } H^{1}(\Omega) \cap C^{0, \alpha}(\bar{\Omega}),
$$

where $\left(\tilde{V}_{1}, \tilde{V}_{2}\right) \in \tilde{M}$ achieves $c_{\infty}$.

Proof. First of all, we notice that, for every $\left(\tilde{v}_{1}, \tilde{v}_{2}\right)$ fixed,

$$
\beta_{1} \leq \beta_{2} \leq+\infty \quad \Longrightarrow \quad J_{\beta_{1}}\left(\tilde{v}_{1}, \tilde{v}_{2}\right) \leq J_{\beta_{2}}\left(\tilde{v}_{1}, \tilde{v}_{2}\right) .
$$

We deduce that $c_{\beta}$ is increasing in $\beta$ and bounded by $c_{\infty}$, thus it converges. If the pair $\left(\tilde{v}_{1, \beta}, \tilde{v}_{2, \beta}\right)$ achieves $c_{\beta}, \beta<+\infty$, then $c_{\beta} \leq c_{\infty}$ implies

$$
\operatorname{both}\left\|\left(\tilde{v}_{1, \beta}, \tilde{v}_{2, \beta}\right)\right\|_{H^{1}}^{2} \leq c_{\infty}+\gamma_{1}+\gamma_{2}, \quad \text { and } \int_{\Omega} \tilde{v}_{1, \beta}^{2} \tilde{v}_{2, \beta}^{2} \leq \frac{c_{\infty}}{\beta} .
$$

We infer the existence of $\left(V_{1}, V_{2}\right)$ such that, up to subsequences, $\tilde{v}_{i, \beta} \rightarrow \tilde{V}_{i}$, weakly in $H^{1}$ and strongly in $L^{p}, p=2,4$. In particular $\left(\tilde{V}_{1}, \tilde{V}_{2}\right) \in M$ and $\tilde{V}_{1} \cdot \tilde{V}_{2} \equiv 0$. We have

$$
c_{\infty} \geq \lim c_{\beta}=\lim J_{\beta}\left(\tilde{v}_{1, \beta}, \tilde{v}_{2, \beta}\right) \geq \liminf \int_{\Omega}\left|\nabla \tilde{v}_{1, \beta}\right|^{2}+\left|\nabla \tilde{v}_{2, \beta}\right|^{2} \geq \int_{\Omega}\left|\tilde{V}_{1}\right|^{2}+\left|\tilde{V}_{2}\right|^{2} \geq c_{\infty} .
$$

Thus $\left(\tilde{V}_{1}, \tilde{V}_{2}\right)$ achieves $c_{\infty}$, and the inequalities above are indeed equalities, proving convergence in $H^{1}$ norm and hence strong $H^{1}$ convergence.

The last thing to prove is the boundedness in $C^{0, \alpha}$ (which will imply convergence in $C^{0, \alpha}$ too, by Ascoli's Theorem). Notice that $\left(\tilde{v}_{1, \beta}, \tilde{v}_{2, \beta}\right)$ satisfies (7), and that $0 \leq \lambda_{i} \leq c_{\infty} / \gamma_{i}$. As a consequence, boundedness of the Hölder seminorm can be obtained as in [25, Theorem 1.1], which provides the same result in the case of Dirichlet boundary conditions: since the proofs in 25 use blow-up arguments, in order to cover the Neumann case one just has to replace odd extensions (from the half-space to $\mathbb{R}^{N}$ ) with even ones. More precisely, this replacement has to be performed in [25, Lemmas 3.4, 3.5, 3.6].

End of the proof of Theorem 1.3. The proof of such theorem easily descends from Lemmas 3.1 , 3.2. when going back to the original unknowns (6). In particular, notice that $\left(\tilde{V}_{1}, \tilde{V}_{2}\right) \in \tilde{M}$ achieves $c_{\infty}$ if and only if $\left(\tilde{V}_{1} / \sqrt{\gamma_{2}}, \tilde{V}_{2} / \sqrt{\gamma_{1}}\right) \in M$ achieves (4). 
To conclude this section, we collect some properties of the minimizers associated to $c_{\infty}$.

Proposition 3.3. Let $\left(\tilde{V}_{1}, \tilde{V}_{2}\right) \in \tilde{M}$ achieve $c_{\infty}$. Then $\tilde{V}_{1} \cdot \tilde{V}_{2} \equiv 0$ and there exist parameters $\Lambda_{i}$ such that

$$
-\Delta\left(\tilde{V}_{1}-\tilde{V}_{2}\right)=\Lambda_{1} \tilde{V}_{1}-\Lambda_{2} \tilde{V}_{2}
$$

(in particular, $\tilde{V}_{1}-\tilde{V}_{2} \in C^{2, \alpha}(\bar{\Omega})$ ).

Furthermore, in dimension $N=1$, let $\Omega=(0,1)$. Then, the unique minimizer is (up to the reflection $x \leftrightarrow 1-x$ )

$$
\begin{aligned}
& \tilde{V}_{1}(x)=\sqrt{\frac{2 \gamma_{2}}{x_{0}}} \cos \left(\frac{\pi}{2 x_{0}} x\right) \cdot \chi_{\left[0, x_{0}\right]}(x), \\
& \tilde{V}_{2}(x)=\sqrt{\frac{2 \gamma_{1}}{1-x_{0}}} \cos \left(\frac{\pi}{2\left(1-x_{0}\right)}(1-x)\right) \cdot \chi_{\left[x_{0}, 1\right]}(x),
\end{aligned}
$$

and $x_{0}=\frac{\sqrt[3]{\gamma_{2}}}{\sqrt[3]{\gamma_{1}}+\sqrt[3]{\gamma_{2}}}$.

Proof. Let

$$
J^{*}(w)=\int_{\Omega}|\nabla w|^{2}, \quad M^{*}=\left\{w \in H^{1}(\Omega): \int_{\Omega}\left(w^{+}\right)^{2}=\gamma_{2}, \int_{\Omega}\left(w^{-}\right)^{2}=\gamma_{1}\right\} .
$$

Then, for component-wise positive pairs, $\left.J_{\infty}\left(\tilde{v}_{1}, \tilde{v}_{2}\right)\right|_{\tilde{M}}=\left.J^{*}\left(\tilde{v}_{1}-\tilde{v}_{2}\right)\right|_{M^{*}}$, and the first part of the proposition follows by the Lagrange multipliers rule (and by standard elliptic regularity).

Turning to the monodimensional case, we have that $\left(\tilde{V}_{1}, \tilde{V}_{2}\right) \in H^{1}(0,1) \times H^{1}(0,1)$ satisfies

$$
-\left(\tilde{V}_{1}-\tilde{V}_{2}\right)^{\prime \prime}=\Lambda_{1} \tilde{V}_{1}-\Lambda_{2} \tilde{V}_{2}, \quad \tilde{V}_{1} \cdot \tilde{V}_{2} \equiv 0, \quad \text { in }(0,1)
$$

with Neumann boundary conditions. By elementary considerations we deduce the existence of (at most countable) disjoint open intervals $I_{i, n}$, with $i=1,2$ and $n \in \mathcal{N}_{i} \subset \mathbb{N}$, such that

$$
V_{i}(x)=\sum_{n \in \mathcal{N}_{i}} a_{i, n} \cos \left(\sqrt{\Lambda_{i}}\left(x-x_{i, n}\right)\right) \cdot \chi_{I_{i, n}}(x),
$$

where

$$
I_{i, n}= \begin{cases}\left(0, \frac{\pi}{2 \sqrt{\Lambda_{i}}}\right) & \text { if } x_{i, n}=0 \\ \left(x_{i, n}-\frac{\pi}{2 \sqrt{\Lambda_{i}}}, x_{i, n}-\frac{\pi}{2 \sqrt{\Lambda_{i}}}\right) & \text { if } x_{i, n} \in\left(\frac{\pi}{2 \sqrt{\Lambda_{i}}}, 1-\frac{\pi}{2 \sqrt{\Lambda_{i}}}\right), \quad \sum_{n} \frac{\pi}{2}\left|I_{i, n}\right| a_{i, n}^{2}=\gamma_{j} . \\ \left(1-\frac{\pi}{2 \sqrt{\Lambda_{i}}}, 1\right) & \text { if } x_{i, n}=1\end{cases}
$$

Now, also the pair defined by

$$
\tilde{W}_{i}=\left.\frac{2 \gamma_{j}}{\pi\left|I_{i, 1}\right| a_{i, 1}^{2}} \tilde{V}_{i}\right|_{I_{i, 1}}
$$

achieves $c_{\infty}$; as a consequence, $\tilde{W}_{1}-\tilde{W}_{2}$ solves (8), while $\tilde{W}_{1}-\tilde{W}_{2} \equiv 0$ outside $I_{1,1} \cup I_{2,1}$. We deduce that both $\mathcal{N}_{i}$ are singleton, and finally that

$$
c_{\infty}=\min \left\{\begin{array}{ll}
w_{1}^{1}\left(w_{1}^{\prime}\right)^{2}+\left(w_{2}^{\prime}\right)^{2}: & \sqrt{\frac{2 \gamma_{2}}{x_{1}}} \cos \left(\frac{\pi}{2 x_{1}} x\right) \cdot \chi_{\left[0, x_{1}\right]}(x) \\
& w_{2}(x)=\sqrt{\frac{2 \gamma_{1}}{1-x_{2}}} \cos \left(\frac{\pi}{2\left(1-x_{2}\right)}(1-x)\right) \cdot \chi_{\left[x_{2}, 1\right]}(x) \\
0<x_{1} \leq x_{2}<1
\end{array}\right\},
$$

whose unique solution can be computed by elementary tools. 


\section{Bifurcation results}

In this section we apply tools from global bifurcation theory in order to prove Theorem 1.4. The main references are the celebrated papers by Rabinowitz [30] and Crandall and Rabinowitz [14], which deal respectively with global bifurcation results for odd eigenvalues, and local ones for simple eigenvalues; for some details about the asymptotic expansions in the latter case, we refer also to [2, Chap. 5]. For the reader's convenience, we recall here the two statements we will apply.

Theorem 4.1 ([30, Thm. 1.3]). Let $E$ be a Banach space, and let $G: \mathbb{R} \times E \rightarrow E$, continuous and compact, be such that

$$
G(\beta, v)=\beta L v+H(\beta, v),
$$

with $L$ linear and compact and $H(\beta, v)=o(\|v\|)$ as $v \rightarrow 0$, uniformly on bounded $\beta$ intervals.

If $\beta^{*}$ is a characteristic value (i.e. $1 / \beta^{*}$ is an eigenvalue) of $L$, having odd multiplicity, then

$$
\mathcal{S}:=\overline{\{(\beta, v): v=G(\beta, v), v \neq 0\}}
$$

possesses a maximal subcontinuum $\mathcal{C}$ such that $\left(\beta^{*}, 0\right) \in \mathcal{C}$, and $\mathcal{C}$ either is unbounded in $E$, or $\left(\beta^{* *}, 0\right) \in \mathcal{C}$, where $\beta^{* *} \neq \beta^{*}$ is another characteristic value of $L$.

Theorem 4.2 (14, Thms. 1.37, 1.18]). Under the assumptions of Theorem 4.1, assume furthermore that $G$ is of class $C^{2}$ and that $\partial_{\beta, v}^{2} G(\beta, 0)=L$.

If $\beta^{*}$ is a simple characteristic value of $L$ and $v^{*} \neq 0$ is such that

$$
\operatorname{Ker}\left(I-\beta^{*} L\right)=\operatorname{span}\left\{v^{*}\right\}, \quad v^{*} \notin \mathrm{R}\left(I-\beta^{*} L\right),
$$

then $\mathcal{S}$ is a continuous curve, locally near $\left(\beta^{*}, 0\right)$, parameterized as

$$
\varepsilon \mapsto(\beta, v)=\left(\beta^{*}+\varphi(\varepsilon), \varepsilon v^{*}+\varepsilon \psi(\varepsilon)\right),
$$

where $\varphi(0)=0, \psi(0)=0$. If $G$ is more regular, then also the above curve is, and one can write higher order expansions (see Remark 4.6).

Among different possible choices, we will apply the above results in the ambient space

$$
E:=\left\{v=\left(v_{1}, v_{2}\right) \in C^{2, \alpha}\left(\bar{\Omega}, \mathbb{R}^{2}\right): \partial_{n} v_{i}=0 \text { on } \partial \Omega\right\} .
$$

Lemma 4.3. The map $G: \mathbb{R} \times E \rightarrow E$ defined as

$$
u=G(\beta, v) \quad \Longleftrightarrow \quad \begin{cases}-\Delta u_{1}+\beta g_{1}\left(v_{2}^{2}\right) u_{1}=\lambda_{1} u_{1} & \\ -\Delta u_{2}+\beta g_{2}\left(v_{1}^{2}\right) u_{2}=\lambda_{2} u_{2} & \text { in } \Omega \\ \int_{\Omega} u_{1}^{2} d x=\int_{\Omega} u_{2}^{2} d x=1, \quad u_{1}, u_{2}>0 & \\ \partial_{n} u_{1}=\partial_{n} u_{2}=0 & \text { on } \partial \Omega,\end{cases}
$$

for suitable $\lambda_{i}$, is (well-defined and) of class $C^{2}$. Moreover it holds

$$
\partial_{v} G(\beta, 1,1)=\beta L,
$$

where

$$
z=L w \quad \Longleftrightarrow \quad \begin{cases}-\Delta z_{1}=-2 g_{1}^{\prime}(1)\left[w_{2}-\int_{\Omega} w_{2}\right] & \\ -\Delta z_{2}=-2 g_{2}^{\prime}(1)\left[w_{1}-\int_{\Omega} w_{1}\right] & \text { in } \Omega \\ \int_{\Omega} z_{1} d x=\int_{\Omega} z_{2} d x=0 & \\ \partial_{n} z_{1}=\partial_{n} z_{2}=0 & \text { on } \partial \Omega .\end{cases}
$$


Proof. The proof is based on standard smooth dependence of simple eigenvalues (and corresponding eigenfunctions) with respect to the potentials, see for instance the book 17. Let us consider the map $F: \mathbb{R} \times E \times E \times \mathbb{R}^{2} \rightarrow C^{0, \alpha}\left(\bar{\Omega}, \mathbb{R}^{2}\right) \times \mathbb{R}^{2}$,

$$
F(\beta, v, u, \lambda):=\left(\begin{array}{c}
-\Delta u_{1}+\beta g_{1}\left(v_{2}^{2}\right) u_{1}-\lambda_{1} u_{1} \\
-\Delta u_{2}+\beta g_{2}\left(v_{1}^{2}\right) u_{2}-\lambda_{2} u_{2} \\
\int_{\Omega} u_{1}^{2} d x-1 \\
\int_{\Omega} u_{2}^{2} d x-1
\end{array}\right)
$$

Let $\beta, v$ be fixed. Then we can uniquely find positive eigenfunctions $u_{i}=u_{i}\left(\beta, v_{j}\right)$ and simple eigenvalues $\lambda_{i}=\lambda_{i}\left(\beta, v_{j}\right)$, such that $F=0$. As a consequence, it is possible to apply the Implicit Function Theorem in order to show that

$$
F(\beta, v, u, \lambda)=0 \quad \Longleftrightarrow \quad(u, \lambda)=\tilde{G}(\beta, v),
$$

with $\tilde{G} \in C^{2}$ (recall that each $g_{i}$ is of class $C^{2}$ ). More precisely, the invertibility of $\partial_{(u, \lambda)} F$ at any of the points above mentioned can be obtained by its injectivity (by Fredholm's Alternative).

Since $G$ is the projection of $\tilde{G}$ on $E$, the first part of the lemma follows. Observing that

$$
\tilde{G}(\beta, 1,1)=(1,1, \beta g(1), \beta g(1)),
$$

also the second part can be proved, by direct calculations.

In order to apply the abstract results, we need to find the eigenvalues of the operator $L$ defined in the previous lemma. In the following, for easier notation, we write

$$
\alpha_{i}=g_{i}^{\prime}(1)>0 \quad(\text { by assumption (2) }) \text {. }
$$

Lemma 4.4. Let $L$ be defined as in (9). Then

$$
\beta^{*} L v^{*}=v^{*}, \quad v^{*} \neq 0, \quad \Longleftrightarrow \quad \beta^{*}=\frac{\mu^{*}}{2 \sqrt{\alpha_{1} \alpha_{2}}}, \quad v^{*}=\left(-\sqrt{\alpha_{1}} \psi^{*}, \sqrt{\alpha_{2}} \psi^{*}\right),
$$

where $\mu^{*}$ is a positive Neumann eigenvalue of $-\Delta$ in $\Omega$ and $\psi^{*}$ a corresponding eigenfunction.

Proof. Recall that $\beta^{*} L v^{*}=v^{*}$ if and only if, for both $i$,

$$
\begin{cases}-\Delta v_{i}^{*}=-2 \beta^{*} \alpha_{i} v_{j}^{*} & \text { in } \Omega \\ \int_{\Omega} v_{i}^{*}=0 & \text { on } \partial \Omega . \\ \partial_{n} v_{i}^{*}=0 & \end{cases}
$$

Setting

$$
\psi_{ \pm}=\sqrt{\alpha_{2}} v_{1} \pm \sqrt{\alpha_{1}} v_{2}
$$

we obtain that the above system is equivalent to

$$
\begin{cases}-\Delta \psi_{ \pm}=\mp 2 \beta^{*} \sqrt{\alpha_{1} \alpha_{2}} \psi_{ \pm} & \text {in } \Omega \\ \int_{\Omega} \psi_{ \pm}=0 & \text { on } \partial \Omega \\ \partial_{n} \psi_{ \pm}=0 & \end{cases}
$$


Hence, if $\beta^{*} \neq 0$, we infer that $\beta^{*}$ is a characteristic value of $L$ if and only if $\psi_{+}=0$ (by the Maximum Principle) and $2 \beta^{*} \sqrt{\alpha_{1} \alpha_{2}}$ is an eigenvalue of $-\Delta$ with zero Neumann boundary conditions. Moreover, the characteristic vector space associated to $\beta^{*}$ is generated by

$$
\left(-\sqrt{\alpha_{1}} \psi^{*}, \sqrt{\alpha_{2}} \psi^{*}\right)
$$

Finally, note that $\beta^{*}=0$ is not a characteristic value of $L$, as $-\Delta \psi=0$ and $\int_{\Omega} \psi=0$ imply that $\psi \equiv 0$.

The last ingredient we need is some control on the behavior of nontrivial solutions.

Lemma 4.5. There exists a constant $C>0$ such that

1. $\mathcal{S} \subset\left\{(\beta, v): \int_{\Omega}|\nabla v|^{2} \leq C \beta\right\} ;$

2. $\mathcal{S} \subset\{(\beta, v): \beta \geq C\}$.

Proof. Recalling Lemma 2.4 we have that, in the present setting,

$$
\int_{\Omega}\left|\nabla v_{i}\right|^{2} \leq \lambda_{i} \leq \beta \int_{\Omega} g_{i}\left(v_{j}^{2}\right) \leq C_{g} \beta,
$$

and the first inclusion follows. Concerning the second one, let by contradiction $\left(\beta_{n}, v_{n}\right)_{n} \subset \mathcal{S}$ be such that $\beta_{n} \rightarrow 0$. Then, by the first inclusion, $v_{n} \rightarrow(1,1)$ in $H^{1}$ and, by elliptic regularity, also in $E$. We deduce that $\beta^{*}=0$ corresponds to a bifurcation point, and therefore $\partial_{v}(\cdot-G(\beta, \cdot))=$ $I-\beta L$ can not be invertible at $\beta=0$, a contradiction.

We are ready to prove our main bifurcation results.

Proof of Theorem 1.4. First of all, let $\mu^{*}$ be a positive Neumann eigenvalue, with odd multiplicity, and

$$
\beta^{*}=\frac{\mu^{*}}{2 \sqrt{\alpha_{1} \alpha_{2}}}
$$

By Lemma 4.4 we are in a position to apply Theorem 4.1 obtaining a nontrivial branch which satisfies one of the alternatives there. Recalling that $\beta=1 / \nu$, we readily have the existence of a nontrivial branch $\mathcal{C}$ in the $(\nu, v)$-space, satisfying all the conditions in (1), with the possible exception of the positivity ones. In view of Lemma 4.5 we have that

$$
\mathcal{C} \subset\left\{(\nu, v): \int_{\Omega}|\nabla v|^{2} \leq \frac{C_{1}}{\nu}, 0<\nu \leq C_{2}\right\} .
$$

Note that, in principle, $\mathcal{C} \cap\{\nu \geq \varepsilon>0\}$ may be unbounded in $C^{2, \alpha}$. Recalling that, in dimension $N \leq 3$, the nolinearities in (1) are Sobolev subcritical, by standard elliptic regularity we have that $H^{1}$ bounds imply $C^{2, \alpha}$ ones, so that unboundedness can happen only as $\nu \rightarrow 0$. The last thing that is left to prove, to complete the first part of the theorem, is that the branch we obtained consists of componentwise positive pairs. This easily follows since, by the Maximum Principle, if the pairs $\left(v_{1, n}, v_{2, n}\right)$ solve (1), with $\nu=\nu_{n}>0$ and $\lambda_{i}=\lambda_{i, n}$, and

$$
v_{i, n} \rightarrow \bar{v}_{i}, \quad \nu_{i, n} \rightarrow \bar{\nu}_{i}, \quad \lambda_{i, n} \rightarrow \bar{\lambda}_{i},
$$

then either $\bar{\nu}=0$ or $\bar{\nu}>0$ and $\bar{v}_{1}, \bar{v}_{2}$ are strictly positive in $\bar{\Omega}$. 
Coming to the second part, let $\mu^{*}$ be a simple positive Neumann eigenvalue. By Lemma 4.4 we have that $\partial_{\beta, v}^{2} G(\beta, 0)=L$. In order to apply Theorem 4.2 , we only have to check the compatibility condition, which in our case writes

$$
\left(-\sqrt{\alpha_{1}} \psi^{*}, \sqrt{\alpha_{2}} \psi^{*}\right) \notin \mathrm{R}\left(I-\beta^{*} L\right)
$$

(here $\psi^{*}$ is an eigenfunction associated to $\mu^{*}=2 \sqrt{\alpha_{1} \alpha_{2}} \beta^{*}$ ). By contradiction, let us assume that $\left(-\sqrt{\alpha_{1}} \psi^{*}, \sqrt{\alpha_{2}} \psi^{*}\right)=\left(I-\beta^{*} L\right) w$, i.e.

$$
\begin{cases}-\Delta w_{i}=-2 \beta^{*} \alpha_{i} w_{j}+(-1)^{i} \mu^{*} \psi^{*} \sqrt{\alpha_{i}} & \text { in } \Omega \\ \int_{\Omega} w_{i}=0 & \text { on } \partial \Omega . \\ \partial_{n} w_{i}=0 & \end{cases}
$$

Reasoning as in the proof of Lemma 4.4, it is easy to prove that $w=0$, and hence $\psi^{*}=0$, a contradiction.

Remark 4.6. If we suppose that $g_{1}$ and $g_{2}$ are smooth, then the branch $\mathcal{S}$ bifurcating from $\left(\beta^{*}, 1,1\right)$ is a smooth curve (at least in a neighborhood of that point), and its parametrization can be made more precise. In order to simplify the following computations, we set

$$
(\beta, v) \in \mathcal{S} \Leftrightarrow 0=\hat{F}(\beta, v, \lambda):=F(\beta, v, v, \beta \lambda)
$$

for some $\lambda \in \mathbb{R}^{2}$, where $F$ is as in (10). Then, $\hat{F}: \mathbb{R} \times E \times \mathbb{R}^{2} \rightarrow C^{0, \alpha}\left(\bar{\Omega}, \mathbb{R}^{2}\right) \times \mathbb{R}^{2}$ is smooth and satisfies

$$
\begin{aligned}
\hat{F}_{v}(\beta, v, \lambda)[w, \ell] & =\left(-\Delta w_{i}+\beta\left(2 g_{i}^{\prime}\left(v_{j}^{2}\right) v_{i} v_{j} w_{j}+g_{i}\left(v_{j}^{2}\right) w_{i}-\ell_{i} v_{i}-\lambda_{i} w_{i}\right), 2 \int_{\Omega} v_{i} w_{i}\right), \\
\hat{F}_{v, \beta}(\beta, v, \lambda)[w, \ell] & =\left(2 g_{i}^{\prime}\left(v_{j}^{2}\right) v_{i} v_{j} w_{j}+g_{i}\left(v_{j}^{2}\right) w_{i}-\ell_{i} v_{i}-\lambda_{i} w_{i}, 0\right), \\
\hat{F}_{v, v}(\beta, v, \lambda)[w, \ell ; h, p] & =\left(\beta\left[4 g_{i}^{\prime \prime}\left(v_{j}^{2}\right) v_{i} v_{j}^{2}+2 g_{i}^{\prime}\left(v_{j}^{2}\right) v_{i}\right] w_{j} h_{j}\right. \\
& \left.+2 \beta g_{i}^{\prime}\left(v_{j}^{2}\right) v_{j} w_{j} h_{i}+2 \beta g_{i}^{\prime}\left(v_{j}^{2}\right) v_{j} w_{i} h_{j}-\beta \ell_{i} h_{i}-\beta p_{i} w_{i}, 2 \int_{\Omega} h_{i} w_{i}\right), \\
\hat{F}_{v, v, v}(\beta, v, \lambda)[w, \ell ; h, p ; z, q] & =\left(\beta\left[8 g_{i}^{\prime \prime \prime} v_{i} v_{j}^{3}+8 g_{i}^{\prime \prime} v_{i} v_{j}+4 g_{i}^{\prime \prime} v_{i} v_{j}\right] w_{j} h_{j} z_{j}+\beta\left[4 g_{i}^{\prime \prime} v_{j}^{2}+2 \beta g_{i}^{\prime}\right] w_{j} h_{j} z_{i}\right. \\
& \left.+\beta\left[4 g_{i}^{\prime \prime} v_{j}^{2}+2 g_{i}^{\prime}\right] w_{j} h_{i} z_{j}+\beta\left[4 g_{i}^{\prime \prime} v_{j}^{2}+2 g_{i}^{\prime}\right] w_{i} h_{j} z_{j}, 0\right) .
\end{aligned}
$$

If $\left(\beta^{*}, 1,1, g_{1}(1), g_{2}(1)\right)$ is a simple bifurcation point, then $\operatorname{Ker}\left(\hat{F}_{v}\right)$ is spanned by the vector $V^{*}=\left(-\sqrt{\alpha_{1}} \psi^{*}, \sqrt{\alpha_{2}} \psi^{*}, 0,0\right)$, and $R\left(\hat{F}_{v}\right)=\{(\Psi, \cdot)=0\}$, where $\Psi=\left(-\sqrt{\alpha_{2}} \psi^{*}, \sqrt{\alpha_{1}} \psi^{*}, 0,0\right)$. Therefore, arguing as in [2, Chap. 5], if we set

$$
A:=\left(\Psi, \hat{F}_{v, \beta}\left[V^{*}\right]\right), \quad B:=\frac{1}{2}\left(\Psi, \hat{F}_{v, v}\left[V^{*}, V^{*}\right]\right), \quad C:=-\frac{1}{6 A}\left(\Psi, \hat{F}_{v, v, v},\left[V^{*}\right]^{3}\right)
$$

where all the derivatives of $\hat{F}$ are evaluated at $\left(\beta^{*}, 1,1, g_{1}(1), g_{2}(1)\right)$, the following expansions hold true

$$
\beta=\beta^{*}-\frac{B}{A} \varepsilon+o(\varepsilon), \quad(\text { if } B \neq 0),
$$

and

$$
\begin{array}{ll}
v=(1,1)+\frac{A}{B}\left(\beta-\beta^{*}\right) \cdot\left(\sqrt{\alpha_{1}} \psi^{*},-\sqrt{\alpha_{2}} \psi^{*}\right)+o\left(\beta-\beta^{*}\right) & \text { if } B \neq 0, \\
v=(1,1) \pm\left(\frac{\beta-\beta^{*}}{C}\right)^{1 / 2} \cdot\left(\sqrt{\alpha_{1}} \psi^{*},-\sqrt{\alpha_{2}} \psi^{*}\right)+O\left(\beta-\beta^{*}\right) & \text { if } B=0, C \neq 0
\end{array}
$$


Note that in the latter case, if $C>0$ (respectively, $C<0$ ) the bifurcating branch emanates on the right (respectively, left) of $\beta^{*}$. In our case, the coefficients $A, B, C$ have the explicit form

$$
\begin{aligned}
& A=-4 g_{1}^{\prime} g_{2}^{\prime} \int\left(\psi^{*}\right)^{2}<0 \\
& B=\beta^{*}\left[2 g_{2}^{\prime \prime} \sqrt{\left(g_{1}^{\prime}\right)^{3}}-2 g_{1}^{\prime \prime} \sqrt{\left(g_{2}^{\prime}\right)^{3}}+3 g_{1}^{\prime} g_{2}^{\prime}\left(\sqrt{g_{1}^{\prime}}-\sqrt{g_{2}^{\prime}}\right)\right] \int\left(\psi^{*}\right)^{3} \\
& C=\frac{\beta^{*}}{-6 A}\left[12 g_{1}^{\prime} g_{2}^{\prime} \sqrt{g_{1}^{\prime} g_{2}^{\prime}}+\sum_{i=1,2}\left(-8\left(g_{j}^{\prime}\right)^{2} g_{i}^{\prime \prime \prime}+12 g_{i}^{\prime \prime}\left(g_{j}^{\prime} \sqrt{g_{i}^{\prime} g_{j}^{\prime}}-\left(g_{j}^{\prime}\right)^{2}\right)\right)\right] \int\left(\psi^{*}\right)^{4},
\end{aligned}
$$

where all the derivatives of $g_{i}$ are evaluated at $s=1$.

We observe that if $N=1$, the bifurcation is always critical, namely $B=0$, as every eigenfunction $\psi^{*}$ satisfies $\int\left(\psi^{*}\right)^{3}=0$. In the variational case, where $g_{i}^{\prime \prime}(1)=g_{i}^{\prime \prime \prime}(1)=0$, the bifurcating branch emanates on the right, namely $(\beta, v) \in \mathcal{S}$ is such that $\beta \geq \beta^{*}$ (and therefore $\nu \leq \nu^{*}$ ), at least in a neighborhood of $\beta^{*}$.

\section{Classification of solutions in dimension $N=1$}

In this section we restrict our attention to the case $\Omega=(0,1) \subset \mathbb{R}$. Consequently, in the following $\left(v_{1}, v_{2}\right)$ denotes a solution of the problem $(i, j=1,2, j \neq i)$

$$
\begin{cases}-\nu v_{i}^{\prime \prime}+g_{i}\left(v_{j}^{2}\right) v_{i}=\lambda_{i} v_{i} & \text { in }(0,1) \\ \int_{0}^{1} v_{i}^{2} d x=1, \quad v>0 & \text { in }[0,1] \\ v_{i}^{\prime}(0)=v_{i}^{\prime}(1)=0 . & \end{cases}
$$

In particular, each $v_{i}$ is $C^{2}([0,1])$, and it has at least an inflection point in $(0,1)$ (just apply Rolle's Theorem to $\left.v_{i}^{\prime}\right)$. Furthermore, $v_{i}^{\prime \prime}(x)$ has the same sign of $g_{i}\left(v_{j}^{2}(x)\right)-\lambda_{i}$, for every $x$.

Lemma 5.1. $v_{i}$ and $v_{j}$ have opposite concavity at 0 and 1. More precisely:

- $g_{i}\left(v_{j}^{2}(0)\right)>\lambda_{i} \Longleftrightarrow g_{j}\left(v_{i}^{2}(0)\right)<\lambda_{j}$;

- $g_{i}\left(v_{j}^{2}(1)\right)>\lambda_{i} \Longleftrightarrow g_{j}\left(v_{i}^{2}(1)\right)<\lambda_{j}$.

Proof. Let us assume, for instance, $g_{i}\left(v_{j}^{2}(0)\right)>\lambda_{i}$ and, by contradiction, $g_{j}\left(v_{i}^{2}(0)\right) \geq \lambda_{j}$ (the other cases are analogous).

Then $v_{i}^{\prime \prime}(0)>0$, and there exists $\xi \in(0,1]$ such that

$$
\left.v_{i}^{\prime \prime}>0 \text { in }[0, \xi), \quad v_{i}^{\prime \prime}(\xi)=0 \text { (and hence } g_{i}\left(v_{j}^{2}(\xi)\right)=\lambda_{i}\right) .
$$

Notice that $\xi<1$, otherwise $v_{i}$ would have no inflection point in $(0,1)$. By convexity and monotonicity we deduce that

$$
x \in(0, \xi] \quad \Longrightarrow \quad v_{i}(x)>v_{i}(0) \quad \Longrightarrow \quad g_{j}\left(v_{i}^{2}(x)\right)-\lambda_{j}>g_{j}\left(v_{i}^{2}(0)\right)-\lambda_{j} \geq 0 .
$$

But then also $v_{j}$ is (convex and) increasing in $[0, \xi]$, so that

$$
g_{i}\left(v_{j}^{2}(\xi)\right)>g_{i}\left(v_{j}^{2}(0)\right)>\lambda_{i},
$$

in contradiction with (11). 
Next we exploit standard uniqueness results for ODEs in order to detect a number of situations in which a considered solution is the trivial one.

Lemma 5.2. Let one of the following condition hold:

1. there exists $\xi \in[0,1]$ such that

$$
g_{1}\left(v_{2}^{2}(\xi)\right)=\lambda_{1}, \quad g_{2}\left(v_{1}^{2}(\xi)\right)=\lambda_{2}, \quad v_{1}^{\prime}(\xi)=v_{2}^{\prime}(\xi)=0
$$

2. there exist $0 \leq x_{1}<x_{2} \leq 1$ such that, for some $i, v_{i}$ is constant in $I=\left[x_{1}, x_{2}\right]$;

3. for some $i, g_{i}\left(v_{j}^{2}(0)\right)=\lambda_{i}$;

4. for some $i, g_{i}\left(v_{j}^{2}(1)\right)=\lambda_{i}$;

Then $\left(v_{1}, v_{2}\right)$ is the trivial solution.

Proof. Under the assumptions of case 1, uniqueness for the Cauchy problem

$$
\left\{\begin{array}{l}
-\nu v_{i}^{\prime \prime}+g_{i}\left(v_{j}^{2}\right) v_{i}=\lambda_{i} v_{i} \quad \text { in }(0,1) \\
v_{i}(\xi)=\sqrt{g_{j}^{-1}\left(\lambda_{j}\right)}, \quad v_{i}^{\prime}(\xi)=0, \quad i=1,2, j \neq i
\end{array}\right.
$$

implies that both $v_{1}$ and $v_{2}$ are constant, and we can conclude exploiting the normalization in $L^{2}(0,1)$.

If 2 holds, the equation for $v_{i}$ implies $g_{i}\left(v_{j}^{2}(\xi)\right)=\lambda_{i}$ on $I$. But then also $v_{j}$ is constant in $I$, forcing $g_{j}\left(v_{i}^{2}(\xi)\right)=\lambda_{j}$. Since both $v_{i}^{\prime}$ and $v_{j}^{\prime}$ are identically zero in $I$, case 1 . applies.

Recalling the Neumann boundary conditions, also cases 3 and 4 can be reduced to 1: indeed, by Lemma [5.1, $g_{i}\left(v_{j}^{2}\right)-\lambda_{i}$ vanishes at one endpoint if and only if $g_{j}\left(v_{i}^{2}\right)-\lambda_{j}$ does.

The following key lemma asserts that between two consecutive maxima of each $v_{i}$ there exists an interval of concavity of $v_{j}$.

Lemma 5.3. Let $0 \leq x_{1}<x_{2} \leq 1$ be such that, for some $i$,

$$
v_{i}^{\prime}\left(x_{1}\right)=v_{i}^{\prime}\left(x_{2}\right)=0, \quad v_{i}^{\prime \prime}\left(x_{1}\right) \leq 0, v_{i}^{\prime \prime}\left(x_{2}\right) \leq 0 .
$$

Then either $\left(v_{1}, v_{2}\right)$ is the trivial solution, or there exists $\xi \in\left(x_{1}, x_{2}\right)$ such that

$$
g_{j}\left(v_{i}^{2}(\xi)\right)<\lambda_{j}
$$

Analogously, if $v_{i}^{\prime}$ vanishes and $v_{i}^{\prime \prime}$ is nonnegative at $x_{1}, x_{2}$ then $g_{j}\left(v_{i}^{2}(\xi)\right)>\lambda_{j}$ for some $\xi \in$ $\left(x_{1}, x_{2}\right)$.

Proof. We have to show that, in case $g_{j}\left(v_{i}^{2}(x)\right) \geq \lambda_{j}$ for every $x \in\left[x_{1}, x_{2}\right]$, then $\left(v_{1}, v_{2}\right)$ is the trivial solution. Under such assumption we have that

$$
\left\{\begin{array}{ll}
v_{j}^{\prime \prime} \geq 0 & \text { in }\left(x_{1}, x_{2}\right) \\
g_{i}\left(v_{j}^{2}\right) \leq \lambda_{i} & \text { at }\left\{x_{1}, x_{2}\right\},
\end{array} \quad \text { so that } g_{i}\left(v_{j}^{2}\right) \leq \lambda_{i} \text { in the whole }\left[x_{1}, x_{2}\right] .\right.
$$

Thus $v_{i}^{\prime \prime} \leq 0$ in $\left[x_{1}, x_{2}\right]$. Since $v_{i}^{\prime}=0$ at $x_{1}$ and $x_{2}$, we obtain that $v_{i}$ is constant in $\left[x_{1}, x_{2}\right]$, and Lemma 5.2 (case 2) applies, concluding the proof.

The above result provides a sharp control on the critical and inflection points of each $v_{i}$, as we show in the next sequence of lemmas. 
Lemma 5.4. If $\left(v_{1}, v_{2}\right)$ is non trivial then both components have only isolated critical points.

Proof. Let by contradiction $\xi \in[0,1]$ be an accumulation point for the set of critical points of $v_{i}$. Of course

$$
v_{i}^{\prime}(\xi)=0
$$

We recall that, for any pair of critical points $x_{1}<x_{2}$, if both $v_{i}^{\prime \prime}\left(x_{1}\right)>0$ and $v_{i}^{\prime \prime}\left(x_{2}\right)>0$ then there exists a third critical point $y_{1} \in\left(x_{1}, x_{2}\right)$ such that $v_{i}^{\prime \prime}\left(y_{1}\right) \leq 0$ (and the same holds for opposite inequalities). Using this fact, it is not difficult to construct two sequences $x_{n} \rightarrow \xi$, $y_{n} \rightarrow \xi$ such that

$$
v_{i}^{\prime}\left(x_{n}\right)=v_{i}^{\prime}\left(y_{n}\right)=0, \quad v_{i}^{\prime \prime}\left(x_{n}\right) \geq 0, v_{i}^{\prime \prime}\left(y_{n}\right) \leq 0 .
$$

Applying repeatedly Lemma 5.3 we deduce the existence of sequences $\bar{\xi}_{n} \rightarrow \xi, \underline{\xi}_{n} \rightarrow \xi$ such that $g_{j}\left(v_{i}^{2}\left(\underline{\xi}_{n}\right)\right)<\lambda_{j}<g_{j}\left(v_{i}^{2}\left(\bar{\xi}_{n}\right)\right)$. This promptly yields

$$
g_{j}\left(v_{i}^{2}(\xi)\right)=\lambda_{j}
$$

Now back to the sequence $\left(x_{n}\right)$, applying Rolle's Theorem we first deduce the existence of a sequence $z_{n} \rightarrow \xi$ such that $0=v_{i}^{\prime \prime}\left(z_{n}\right)=g_{i}\left(v_{j}^{2}\left(z_{n}\right)\right)-\lambda_{i}$, implying

$$
g_{i}\left(v_{j}^{2}(\xi)\right)=\lambda_{i}
$$

and then of a sequence $z_{n}^{\prime} \rightarrow \xi$ with

$$
0=v_{j}^{\prime}\left(z_{n}^{\prime}\right) \rightarrow v_{j}^{\prime}(\xi)
$$

Summing up, we are in a position to apply Lemma 5.2 (Case 1), obtaining that $\left(v_{1}, v_{2}\right)$ is trivial, a contradiction.

Lemma 5.5. Let $x_{0} \in[0,1]$ be a point of local minimum for $v_{i}$. Then either $\left(v_{1}, v_{2}\right)$ is the trivial solution, or

$$
g_{j}\left(v_{i}^{2}\left(x_{0}\right)\right)<\lambda_{j}, \quad g_{i}\left(v_{j}^{2}\left(x_{0}\right)\right)>\lambda_{i}
$$

(in particular, it is non degenerate). An analogous statement (with reverse inequalities) holds for local maxima.

Proof. If $x_{0}=0$ or $x_{0}=1$, then the statement is a consequence of Lemma 5.1. Otherwise, since $x_{0}$ is an isolated critical point, it is a strict minimum, and the following points are well defined:

$$
x_{1}=\inf \left\{x \in\left[0, x_{0}\right): v_{i}^{\prime}<0 \text { in }\left(x, x_{0}\right)\right\}, \quad x_{2}=\sup \left\{x \in\left(x_{0}, 1\right]: v_{i}^{\prime}>0 \text { in }\left(x_{0}, x\right)\right\} .
$$

Then $x_{1}, x_{2}$ satisfy the assumptions of Lemma 5.3, providing the existence of $\xi \in\left(x_{1}, x_{2}\right)$ such that

$$
g_{j}\left(v_{i}^{2}\left(x_{0}\right)\right)=\min _{\left[x_{1}, x_{2}\right]} g_{j}\left(v_{i}^{2}\right) \leq g_{j}\left(v_{i}^{2}(\xi)\right)<\lambda_{j}
$$

which is the first inequality required.

On the other hand, since $x_{0}$ is an isolated strict minimum we have that $g_{i}\left(v_{j}^{2}\left(x_{0}\right)\right) \geq \lambda_{i}$ in a neighborhood of $x_{0}$. Since the last inequality implies that $v_{j}$ is strictly concave in a neighborhood of $x_{0}$, we deduce also the second (strict) inequality.

Lemma 5.6. If $\left(v_{1}, v_{2}\right)$ is not the trivial solution, then any critical point of each component is non degenerate. 
Proof. Let us assume by contradiction that $\xi$ is a degenerate critical point of $v_{i}$. By Lemmas 5.4, 5.5 we have that $\xi \in(0,1)$ is an isolate inflection point. Therefore

$$
v_{i}^{\prime}(\xi)=0, \quad g_{i}\left(v_{j}^{2}(\xi)\right)=\lambda_{i}
$$

and $\xi$ is a local extremum for $v_{j}$. But then Lemma 5.5 applies again, implying that either $g_{i}\left(v_{j}^{2}(\xi)\right)>\lambda_{i}$ or $g_{i}\left(v_{j}^{2}(\xi)\right)<\lambda_{i}$, a contradiction.

Lemma 5.7. Let $\left(v_{1}, v_{2}\right)$ be non trivial, and $x_{1}<x_{2}$ be such that, for some $i$,

$$
v_{i}^{\prime}\left(x_{1}\right)=v_{i}^{\prime}\left(x_{2}\right)=0, \quad v_{i}^{\prime}>0 \text { in }\left(x_{1}, x_{2}\right) .
$$

Then both $v_{i}$ and $v_{j}$ have exactly one inflection point in $\left[x_{1}, x_{2}\right]$. An analogous statement holds for the opposite monotonicity.

Proof. By Lemma [5.5 we immediately deduce the existence of $\xi \in\left(x_{1}, x_{2}\right)$ such that

$$
v_{j}^{\prime \prime}<0 \text { in }\left[x_{1}, \xi\right), \quad v_{j}^{\prime \prime}>0 \text { in }\left(\xi, x_{2}\right]
$$

and $v_{j}$ has exactly one inflection point in $\left[x_{1}, x_{2}\right]$.

On the other hand, the inflection points of $v_{i}$ are the solutions of

$$
g_{j}\left(v_{i}^{2}(x)\right)=\lambda_{j}, \quad x \in\left[x_{1}, x_{2}\right] .
$$

Since $g_{j}\left(v_{i}^{2}\left(x_{1}\right)\right)>\lambda_{j}$ and $g_{j}\left(v_{i}^{2}\left(x_{2}\right)\right)<\lambda_{j}$ (and again by Lemma 5.5), equation (13) has an odd number of solutions. On the other hand, taking into account (12), equation (13) has at most one solution in $\left[x_{1}, \xi\right]$ and one in $\left[\xi, x_{2}\right]$, respectively.

Collecting the previous results we have the following characterization of nontrivial solutions.

Proposition 5.8. If $\left(v_{1}, v_{2}\right)$ is not the trivial solution, then there exists $k \in \mathbb{N}$ such that both $v_{1}$ and $v_{2}$ have exactly $k$ critical points, all non degenerate, and $k+1$ isolated inflection points in $(0,1)$.

Proof. Let $n_{i}$ denote the number of critical points of $v_{i}$ in $(0,1)$ (they are well defined by Lemma 5.4), and $m_{i}$ the number of inflection points. By Lemma 5.7 we have that $n_{i}+2=m_{i}=m_{j}$, and the claim follows.

We are ready to conclude the proof of the main result of this section.

Proof of Theorem 1.7 (first part). First of all let $\mathcal{C} \subset \mathcal{S}$ be a continuum of nontrivial solutions, and

$$
\mathcal{C} \ni\left(\nu_{n}, v_{1, n}, v_{2, n}\right) \rightarrow\left(\bar{\nu}, \bar{v}_{1}, \bar{v}_{2}\right), \quad \text { as } n \rightarrow+\infty .
$$

Using Proposition 5.8, it is not difficult to prove that, if the number of interior critical points of each $v_{i, n}$ is constant, equal to $k$, then

- either $\bar{\nu}=0$;

- or $\bar{\nu}>0$ and $\left(\bar{v}_{1}, \bar{v}_{2}\right)$ is the trivial solution;

- or $\bar{\nu}>0$ and each $\bar{v}_{i}$ has exactly $k$ interior critical points. 
Now recall that, being $N=1$ and $\Omega=(0,1)$, the Neumann eigenvalues and eigenfunction of $-\partial_{x x}^{2}$ have the form

$$
\mu_{k}=(k \pi)^{2}, \quad \psi_{k}=A \cos (k \pi x)(A \neq 0), \quad k \in \mathbb{N},
$$

and every eigenvalue $\mu_{k}$ is simple. Applying Theorem 1.4 we have the existence, for every $k \geq 1$, of continua $\mathcal{C}_{k} \subset \mathcal{S}$ which consist, locally near $\left(\mu_{k}, 1,1\right)$, of pairs having exactly $k-1$ critical points (by the local parameterization, because also $\psi_{k}$ has exactly $k-1$ critical points). The initial argument tells that each $\mathcal{C}_{k}$ is characterized by the number of critical points of its components, so that two of them cannot meet, and each of them is unbounded in the sense of Theorem 1.4 (since we are in dimension $N=1 \leq 3$ ).

We are only left to prove segregation: this is the object of the next section.

\section{$6 \quad$ Segregation in dimension $N=1$}

As we already mentioned (see Remark 1.6), we can not expect that all arbitrary families of nontrivial solutions segregate. Nonetheless, restricting our attention to $\mathcal{C}_{k}$ as in Theorem 1.7 for some fixed $k$, we can obtain more precise results.

In the following, we focus on $\left(\nu_{n}, v_{1, n}, v_{2, n}\right) \subset \mathcal{C}_{k}$, a sequence of solutions of (1), with $\nu=$ $\nu_{n}>0$ and $\lambda_{i}=\lambda_{i, n}$, whose components have exactly $k-1$ critical points in $(0,1)$, all nondegenerate. For easier notation, we will drop the subscript $n$ throughout the proofs, except when some confusion may arise; in particular, properties of

$$
v_{1}, v_{2}, \lambda_{1}, \lambda_{2} \quad \text { as } \nu \rightarrow 0,
$$

are those of the considered sequence, when $\nu_{n} \rightarrow 0$ as $n \rightarrow+\infty$.

As a first step, we want to rule out the possibility that the branch "collapses" to the trivial solution as $\nu \rightarrow 0$.

Proposition 6.1. Suppose that

$$
v_{1, n} \rightarrow 1, \quad v_{2, n} \rightarrow 1, \quad \nu_{n} \rightarrow \bar{\nu},
$$

where the convergence is uniform in $[0,1]$. Then, $\lambda_{i, n} \rightarrow g_{i}(1)$ and $\bar{\nu}>0$.

Proof. The proof will be carried out in three steps, and considering the system solved by $u_{i}:=$ $v_{i}-1$, which is

$$
\left\{\begin{array}{l}
-\nu u_{1}^{\prime \prime}=\left(\lambda_{1}-G_{1}\left(1+u_{2}\right)\right)\left(1+u_{1}\right), \quad \text { in }(0,1) \\
-\nu u_{2}^{\prime \prime}=\left(\lambda_{2}-G_{2}\left(1+u_{1}\right)\right)\left(1+u_{2}\right), \\
u_{1}^{\prime}=u_{2}^{\prime}=0 \text { at }\{0,1\}, \\
\int_{0}^{1}\left(1+u_{1}\right)^{2}=\int_{0}^{1}\left(1+u_{2}\right)^{2}=1,
\end{array}\right.
$$

where we have set $G_{i}(t):=g_{i}\left(t^{2}\right)$ for all $t \geq 0$. Note that $u_{i} \rightarrow 0$ uniformly in $[0,1]$.

Without loss of generality, we set $\left(\bar{x}=\bar{x}_{n}\right)$

$$
M:=\max _{i=1,2, x \in[0,1]}\left|u_{i}(x)\right|=u_{1}(\bar{x}) .
$$

$\underline{\text { Step 1. }}\left|\lambda_{i}-G_{i}(1)\right| / M \rightarrow 0$ as $n \rightarrow \infty$. Indeed, note first that $\int_{0}^{1}\left(1+u_{i}\right)^{2}=1$ implies that

$$
\int_{0}^{1} u_{i}=-\frac{1}{2} \int_{0}^{1} u_{i}^{2}
$$


Moreover, a Taylor expansion in the equations of (14) gives

$$
-\nu u_{i}^{\prime \prime}=\left(\lambda_{i}-G_{i}(1)-G_{i}^{\prime}(1) u_{j}-\frac{G_{i}^{\prime \prime}(\xi)}{2} u_{j}^{2}\right)\left(1+u_{i}\right),
$$

where $\xi$ is a bounded function in $(0,1)$ (uniformly with respect to $n$ ). By integrating the equation and using the boundary conditions we obtain

$$
\left(\lambda_{i}-G_{i}(1)\right) \int_{0}^{1}\left(1+u_{i}\right)=G_{i}^{\prime}(1) \int_{0}^{1} u_{j}+G_{i}^{\prime}(1) \int_{0}^{1} u_{i} u_{j}+\int_{0}^{1} \frac{G_{i}^{\prime \prime}(\xi)}{2} u_{j}^{2}\left(1+u_{i}\right) .
$$

Hence, using (15),

$$
\left|\lambda_{i}-G_{i}(1)\right| \int_{0}^{1}\left(1+u_{i}\right) \leq \frac{G_{i}^{\prime}(1)}{2} \int_{0}^{1} u_{j}^{2}+G_{i}^{\prime}(1) \int_{0}^{1}\left|u_{i}\right|\left|u_{j}\right|+\int_{0}^{1} \frac{G_{i}^{\prime \prime}(\xi)}{2} u_{j}^{2}\left(1+u_{i}\right)
$$

which leads to the assertion, as $\int_{0}^{1}\left(1+u_{i}\right) \rightarrow 1,\left|u_{i}\right|,\left|u_{j}\right| \leq M$ in $[0,1]$ and $M \rightarrow 0$. The first conclusion of the proposition also follows, as $G_{i}(1)=g_{i}(1)$.

Step 2. Assume by contradiction that $\bar{\nu}=0$. We proceed with a blow-up analysis, setting

$$
\tilde{u}_{i}(x)=\frac{1}{M} u_{i}(\sqrt{\nu} x+\bar{x}) \quad \forall x \in\left(-\frac{\bar{x}}{\sqrt{\nu}}, \frac{1-\bar{x}}{\sqrt{\nu}}\right)=: \widetilde{\Omega}_{n}
$$

We have that $\left|\tilde{u}_{i}\right| \leq 1$ in $\widetilde{\Omega}_{n}$ and $\tilde{u}_{1}(0)=1$. Moreover, $\tilde{u}_{i}$ solves

$$
-\tilde{u}_{i}^{\prime \prime}=\left(\frac{\lambda_{i}-G_{i}(1)}{M}-\left(G_{i}^{\prime}(1)+o(1)\right) \tilde{u}_{j}\right)\left(1+u_{i}\right) \quad \text { in } \widetilde{\Omega}_{n} .
$$

Note that (up to subsequences)

$$
\widetilde{\Omega}_{n} \rightarrow \widetilde{\Omega}_{\infty}:= \begin{cases}{[\bar{X},+\infty)} & \text { if }-\frac{\bar{x}}{\sqrt{\nu}} \rightarrow \bar{X}<+\infty \\ (-\infty, \bar{X}] & \text { if } \frac{1-\bar{x}}{\sqrt{\nu}} \rightarrow \bar{X}<+\infty \\ \mathbb{R} & \text { otherwise. }\end{cases}
$$

Using the equation (twice) and the uniform boundedness of $\tilde{u}_{i}$ on $\widetilde{\Omega}_{n}$, we argue that $\tilde{u}_{i}^{\prime \prime \prime}$ is bounded on compact subsets of $[0, \infty)$, uniformly as $n \rightarrow \infty$. Hence, $\tilde{u}_{i} \rightarrow \widetilde{U}_{i} \in C^{2}\left(\widetilde{\Omega}_{\infty}\right)$ locally in $C^{2, \alpha}$ where $\widetilde{U}_{i}$ has at most $k$ intervals of monotonicity and solve, in $\widetilde{\Omega}_{\infty}$,

$$
\left\{\begin{array}{l}
\widetilde{U}_{1}^{\prime \prime}=G_{1}^{\prime}(1) \widetilde{U}_{2} \\
\widetilde{U}_{2}^{\prime \prime}=G_{2}^{\prime}(1) \widetilde{U}_{1},
\end{array}\right.
$$

in view of the conclusion of Step 1 . Note that, in case $\widetilde{\Omega}_{\infty} \neq \mathbb{R}$, we can use the Neumann conditions in order to extend $\widetilde{U}_{i}$ by even reflection around $\bar{X}$, in such a way that $\widetilde{U}_{1}, \widetilde{U}_{2}$ solve (16) in the whole $\mathbb{R}$.

Step 3. To reach a contradiction we are going to show that system (16) does not admit nontrivial bounded solutions having a finite number of oscillations (recall that $\widetilde{U}_{1}(0)=1$ ). We can reason as in Section 4 setting

$$
W_{ \pm}=\sqrt{\alpha_{2}} \widetilde{U}_{1} \pm \sqrt{\alpha_{1}} \widetilde{U}_{2}
$$


and obtaining a decoupled system:

$$
\left\{\begin{array}{l}
W_{+}^{\prime \prime}=\sqrt{\alpha_{1} \alpha_{2}} W_{+} \\
W_{-}^{\prime \prime}=-\sqrt{\alpha_{1} \alpha_{2}} W_{-}
\end{array}\right.
$$

Therefore, since $W_{+}$is bounded it must be constant and identically zero. We deduce that $\widetilde{U}_{1}$, $\widetilde{U}_{2}$ are proportional, so that

$$
\widetilde{U}_{1}^{\prime \prime}=-\sqrt{\alpha_{1} \alpha_{2}} \widetilde{U}_{1},
$$

which forces $\widetilde{U}_{1} \equiv 0$ (since it has at most $2 k$ monotonicity intervals in $\mathbb{R}$ ).

Next we turn to the case in which $\left\|v_{i}\right\|_{L^{\infty}}$ is uniformly bounded along the sequence, for both $i$. To treat such case we need the following Liouville-type result.

Lemma 6.2. Let $V_{i} \in C^{2}(\mathbb{R}), 0 \leq V_{i} \leq M, \Lambda_{i} \geq 0$ be such that

$$
-V_{i}^{\prime \prime}=\left(\Lambda_{i}-g_{i}\left(V_{j}^{2}\right)\right) V_{i} \quad \text { in } \mathbb{R} .
$$

If both $V_{i}$ have at most a finite number of monotonicity intervals, then one of the following holds:

1. either $V_{1} \equiv 0, \Lambda_{2}=0$,

2. or $V_{2} \equiv 0, \Lambda_{1}=0$,

3. or $V_{1} \equiv V_{2} \equiv 0$,

4. or $g_{i}\left(V_{j}^{2}\right) \equiv \Lambda_{i}, i=1,2$.

Proof. First of all, we can reason as in Lemma 5.2 to show that, if some $V_{i}$ is constant in an interval, then $\left(V_{1}, V_{2}\right)$ is constant in $\mathbb{R}$, and as a consequence we always fall in one of the above cases. Secondly, assume that some $\Lambda_{i}=0$ : then $V_{i}$ is constant, and again the lemma follows by elementary considerations.

We are left to deal with the case $\Lambda_{1}, \Lambda_{2}>0$ and $V_{1}, V_{2}$ non constant and strictly positive. Since both $V_{i}$ have a finite number of monotonicity intervals, the equations imply that they also have a finite number of inflection points (and they have at least one, since they are bounded in $\mathbb{R})$. We deduce the existence of $a \in \mathbb{R}$ such that, say,

$$
V_{1}^{\prime}, V_{2}^{\prime}, V_{1}^{\prime \prime}, V_{2}^{\prime \prime} \text { do not change sign in }(a,+\infty) \text {. }
$$

In particular, the limits $V_{i}(+\infty)$ exist and $V_{i}^{\prime}(+\infty)=V_{i}^{\prime \prime}(+\infty)=0$.

Assume that $V_{i}^{\prime} \geq 0$ for $x>a$, so that $V_{i}^{\prime \prime} \leq 0$ in the same interval. Then $V_{i}(+\infty)>0$, and

$$
g_{i}\left(V_{j}^{2}(x)\right) \leq \Lambda_{i} \text { in }(a,+\infty), \quad g_{i}\left(V_{j}^{2}(+\infty)\right)=\Lambda_{i}-\frac{V_{i}^{\prime \prime}(+\infty)}{V_{i}(+\infty)}=\Lambda_{i},
$$

so that also $V_{j}^{\prime}$ is non negative.

Now we can lower $a$ in such a way that, say,

$$
V_{1}^{\prime \prime}(a)=0 .
$$

If $V_{1}^{\prime} \leq 0$ for $x>a$, we deduce that also $V_{2}^{\prime} \leq 0$ in the same interval. But then $\Lambda_{1}-g_{1}\left(V_{2}^{2}\right)$ is increasing, and $V_{1}^{\prime \prime} \leq 0$ for $x>a$, a contradiction since $V_{1}$ is decreasing and bounded.

On the other hand, let $V_{1}^{\prime} \geq 0$ for $x>a$. Then $V_{1}(+\infty)>0$, and

$$
g_{1}\left(V_{2}^{2}(a)\right)=\Lambda_{1}=g_{1}\left(V_{2}^{2}(+\infty)\right)-\frac{V_{1}^{\prime \prime}(+\infty)}{V_{1}(+\infty)}=g_{1}\left(V_{2}^{2}(+\infty)\right),
$$

forcing $V_{2}$ to be constant in $[a,+\infty)$, again a contradiction. 
Using the previous result, we can show that uniform $L^{\infty}$ bounds imply segregation.

Lemma 6.3. Assume that $\left\|v_{i, n}\right\|_{\infty} \leq C$ for both $i$. Then, up to subsequences,

$$
\lambda_{i, n} \rightarrow 0, \quad i=1,2,
$$

as $\nu_{n} \rightarrow 0$.

Proof. Let us assume by contradiction that, for instance, $\lambda_{1} \nrightarrow \rightarrow 0$. We choose a sequence $\left(x_{1, n}\right)_{n} \subset$ $[0,1]$ such that (omitting the subscript $n$ )

$$
v_{1}\left(x_{1}\right):=\max _{[0,1]} v_{1} \geq 1, \quad \text { and put } \tilde{v}_{i}(x):=v_{i}\left(x_{1}+x \sqrt{\nu}\right) .
$$

Then, $\tilde{v}_{i}$ solves

$$
-\tilde{v}_{i}^{\prime \prime}(x)=\left(\lambda_{i}-g_{i}\left(\tilde{v}_{j}^{2}(x)\right)\right) \tilde{v}_{i}(x) \quad \text { in }\left(-x_{1} \nu^{-1 / 2},\left(1-x_{1}\right) \nu^{-1 / 2}\right),
$$

$\left\|\tilde{v}_{i}\right\|_{\infty} \leq C, \tilde{v}_{i} \geq 0$. The equations and (5) guarantee local $C^{3}$ boundedness of $\tilde{v}_{i}$, thus, up to subsequences, $\tilde{v}_{i} \rightarrow V_{i}$ locally in $C^{2}$. Moreover, $\lambda_{i} \rightarrow \Lambda_{i} \geq 0$. We argue that, possibly up to an even extension, each $V_{i}$ has at most $2 k$ intervals of monotonicity and

$$
-V_{i}^{\prime \prime}=\left(\Lambda_{i}-g_{i}\left(V_{j}^{2}\right)\right) V_{i} \quad \text { in } \mathbb{R} .
$$

Then, Lemma 6.2 applies, but since $V_{1}(0) \geq 1$ and $\Lambda_{1}>0$, we deduce that

$$
g_{i}\left(v_{j}^{2}\left(x_{1}\right)\right)-\lambda_{i} \rightarrow 0 \text { for both } i,
$$

and also $\lambda_{2} \nrightarrow \rightarrow 0\left(\right.$ as $\left.g_{2}\left(v_{1}^{2}\left(x_{1}\right)\right) \geq g_{2}(1)>0\right)$. We can implement the same argument using

$$
v_{1}\left(x_{2}\right):=\min _{[0,1]} v_{1} \leq 1, \quad \text { and } \tilde{w}_{i}(x):=w_{i}\left(x_{2}+x \sqrt{\nu}\right) .
$$

Passing to the limit (we keep the same sequence $\lambda_{i} \rightarrow \Lambda_{i}>0$ as before), and recalling Lemma 5.5. we have that $W_{2}(0)>0$ and then

$$
g_{i}\left(v_{j}^{2}\left(x_{2}\right)\right)-\lambda_{i} \rightarrow 0 \text { for both } i .
$$

Combining (17) and (18), we deduce that $v_{1} \rightarrow 1$ uniformly on [0,1].

Now, since also $\Lambda_{2}>0$, we can exchange the role of $v_{1}$ and $v_{2}$, obtaining that $v_{2} \rightarrow 1$ too, in contradiction with Proposition 6.1.

We are left to deal with the case of $\max _{[0,1]}\left(v_{1, n}+v_{2, n}\right) \rightarrow+\infty$, namely when $v_{1}$ or $v_{2}$ are not bounded uniformly in $n$. To treat this situation we need to exploit the finite number of maxima of each component along $\mathcal{C}_{k}$, as enlighten in the following lemma (for convenience we write explicitely the dependence on $n$ ).

Lemma 6.4. Let $\max _{[0,1]}\left(v_{1, n}+v_{2, n}\right) \rightarrow+\infty$. There exist and index $i$, constants $C, \delta>0$ (independent of $n$ ), and a sequence of intervals $I_{n} \subset[0,1]$ such that, up to subsequences:

$$
\begin{aligned}
& \left|I_{n}\right|=\delta \\
& \max _{I_{n}} v_{i, n}=\max _{\partial I_{n}} v_{i, n} \rightarrow+\infty \\
& \max _{I_{n}} v_{j, n} \leq C .
\end{aligned}
$$


Proof. Let

$Z_{n}:=\left\{z \in[0,1]: z\right.$ is a local maximum for $v_{i, n}$, for some $i$, and $\left.v_{i, n}(z) \rightarrow+\infty\right\}$.

Since we are considering elements of $\mathcal{C}_{k}$, we have that

$$
Z_{n}=\left\{z_{l, n}\right\}_{l=1, \ldots, h}, \quad \mathrm{z}_{1, n}<\cdots<z_{h, n}, \quad h \leq k+1
$$

(recall that, by Lemma 5.5. if $z$ is a local maximum for $v_{i}$ then $\left.g_{i}\left(v_{j}^{2}(z)\right) \leq \lambda_{i}\right)$. Up to subsequences, we can assume that each $z_{l, n}$ is a maximum for some $v_{i, n}$, with $i$ independent of $n$; furthermore we can assume that, for each $l, z_{l, n} \rightarrow z_{l} \in[0,1]$. We distinguish three cases.

Case 1: for some $l, z_{l}<z_{l+1}$. We choose $i$ so that $z_{l, n}$ is a local maximum for $v_{i, n}$ and

$$
2 \delta=z_{l+1}-z_{l}, \quad I_{n}=\left[z_{l, n}, z_{l, n}+\delta\right] .
$$

By construction, neither $v_{i, n}$ nor $v_{j, n}$ can have interior maxima which go to infinity; therefore the required properties for $\max _{I_{n}} v_{i, n}$ follow from the fact that $v_{i, n}\left(z_{l, n}\right) \rightarrow+\infty$, while those for $\max _{I_{n}} v_{j, n}$ descend again by Lemma 5.5.

Case 2: $z_{1}=\cdots=z_{h} \neq 1$. One can reason as above, by choosing $i$ so that $z_{h, n}$ is a local maximum for $v_{i, n}$ and

$$
2 \delta=1-z_{h}, \quad I_{n}=\left[z_{h, n}, z_{h, n}+\delta\right] .
$$

Case 3: $z_{1}=\cdots=z_{h} \neq 0$. We can choose $i$ so that $z_{1, n}$ is a local maximum for $v_{i, n}$ and

$$
2 \delta=z_{1}, \quad I_{n}=\left[z_{1, n}-\delta, z_{1, n}\right] .
$$

The last tool we need is the following standard comparison lemma.

Lemma 6.5 ([12, Lemma 4.4]). Suppose that $u \in C^{2}(a, b) \cap C([a, b])$ satisfies

$$
-u^{\prime \prime}(x) \leq-M u(x), \quad 0 \leq u(x) \leq A, \quad \text { in }(a, b)
$$

for some $A, M>0$. Then, for every $0<\delta<(b-a) / 2$,

$$
u(x) \leq 2 A e^{-\delta \sqrt{M}} \quad \text { in }[a+\delta, b-\delta] .
$$

Proof. By comparison with the solution of $-w^{\prime \prime}=-M w$ in $(a, b), w(a)=w(b)=A$.

Remark 6.6. By even reflection, we have that if $u$ is as in Lemma 6.5 and furthermore $u^{\prime}(a)=0$, then the estimate holds on any $\left[a, b^{\prime}\right] \subset[a, b)$, choosing $\delta=b-b^{\prime}$.

We are in a position to prove that segregation occurs also when some $v_{i}$ is unbounded, thus completing the proof of Theorem 1.7

Lemma 6.7. Let $\max _{[0,1]}\left(v_{1, n}+v_{2, n}\right) \rightarrow+\infty$. Then (up to subs.) $\lambda_{i, n} \rightarrow 0$, for some $i$ (and the corresponding $v_{i, n}$ is not uniformly bounded).

Proof. Let $i, I_{n}=:\left[z_{n}, z_{n}+\delta\right]$ be as in Lemma 6.4. We can assume, w.l.o.g.,

$$
\max _{I_{n}} v_{i, n}=v_{i, n}\left(z_{n}\right) \rightarrow+\infty .
$$

We define the blow-up sequences

$$
\begin{aligned}
& \tilde{v}_{i, n}(x):=\frac{1}{v_{i, n}\left(z_{n}\right)} v_{i, n}\left(z_{n}+x \sqrt{\nu_{n}}\right) \\
& \tilde{v}_{j, n}(x):=v_{j, n}\left(z_{n}+x \sqrt{\nu_{n}}\right) .
\end{aligned}
$$


Then, $\tilde{v}_{i, n}=\tilde{v}_{i}$ solves

$$
-\tilde{v}_{i}^{\prime \prime}=\left(\lambda_{i}-g_{i}\left(\tilde{v}_{j}^{2}\right)\right) \tilde{v}_{i}
$$

in $\left(0, \delta \nu^{-1 / 2}\right), 0 \leq \tilde{v}_{i} \leq 1$ and $\tilde{v}_{i}(0)=1$. Also $\lambda_{i}-g_{i}\left(\tilde{v}_{j}^{2}\right)$ is uniformly bounded in $\left[0, \delta \nu^{-1 / 2}\right]$, by Lemmas 2.4 and 6.4. Since both $\tilde{v}_{i}$ and $\tilde{v}_{i}^{\prime \prime}$ are uniformly bounded on compact sets, we deduce that also $\tilde{v}_{i}^{\prime}$ is bounded, and there exists $V \in C^{1}([0,+\infty))$ such that $v_{i} \rightarrow V$ in $C^{1}([a, b])$, for every $[a, b] \subset[0,+\infty)$.

We claim that, if $V>0$ in $\left[a^{\prime}, b^{\prime}\right] \subset(0,+\infty)$, then $\tilde{v}_{j} \rightarrow 0$ uniformly in $\left[a^{\prime}, b^{\prime}\right]$. Indeed, let $(a, b) \supset\left[a^{\prime}, b^{\prime}\right]$ be such that $V \geq \eta>0$ in $(a, b)$. We deduce that, in such interval,

$$
-\tilde{v}_{j}^{\prime \prime}=\left(\lambda_{j}-g_{j}\left(v_{i}^{2}(z) \tilde{v}_{i}^{2}\right)\right) \tilde{v}_{j} \leq\left(\lambda_{j}-C_{g}^{-1} v_{i}^{2}(z) \frac{1}{2} V_{i}^{2}\right) \tilde{v}_{j} \leq-C^{2} v_{i}^{2}(z) \tilde{v}_{j},
$$

where $C>0$ depends on $\eta$ and $C_{g}$. Lemma 6.5] applies, yielding

$$
0 \leq \tilde{v}_{j} \leq C_{1} e^{-C_{2} v_{i}(z)} \rightarrow 0 \quad \text { in }\left[a^{\prime}, b^{\prime}\right],
$$

as $C_{2}>0$ and $v_{i}(z) \rightarrow+\infty$.

Now, let $\lambda_{i} \rightarrow \Lambda \geq 0$. We can pass to the limit in the equation of $\tilde{v}_{i}$, deducing that

$$
\left\{\begin{array}{l}
V \in C^{1}([0,+\infty)), \quad 0 \leq V \leq 1, \\
V>0 \Longrightarrow-V^{\prime \prime}=\Lambda V \\
V(0)=1
\end{array}\right.
$$

Let $[0, a), a \leq+\infty$, be the maximal interval containing 0 in which $V>0$. If $a<+\infty$, by convexity we obtain that $V(a)=0$ and $V^{\prime}(a)<0$, a contradiction since $V(x)$ must be non negative also for $x>a$. Therefore $a=+\infty$ and $V$ is a bounded, concave function on $\mathbb{R}^{+}$, i.e. $V \equiv 1$ and $\Lambda=0$.

End of the proof of Theorem 1.7. Taking into account Corollary 2.5] the last part of the theorem follows from Lemmas 6.3 and 6.7

\section{Further properties of the first branch}

To conclude, we complete the analysis started in Section 6 by restricting our attention to the first bifurcation branch $\mathcal{C}_{1}$. Since $k=1$, such branch consists of monotone solutions, and for concreteness we assume that the sequence we are considering is such that $v_{1, n}$ is decreasing and $v_{2, n}$ is increasing (and $\nu_{n} \rightarrow 0$ as $n \rightarrow \infty$ ). As before, we will omit the subscript $n$, when no confusion arises. We denote by $\xi_{1, n}, \xi_{2, n} \in(0,1)$ the unique inflection points of the considered pair:

$$
-v_{1, n}^{\prime}\left(\xi_{1, n}\right)=\max _{[0,1]}\left|v_{1, n}^{\prime}(x)\right|, \quad v_{2, n}^{\prime}\left(\xi_{2, n}\right)=\max _{[0,1]}\left|v_{2, n}^{\prime}(x)\right| .
$$

A number of (rather elementary) a priori estimates can be deduced from the monotonicity of the components. We collect them in the following three lemmas. 
Lemma 7.1. The following inequalities hold

$$
\begin{aligned}
& v_{1}^{2}(x) \leq \frac{1}{x} \quad \forall x>0, \quad v_{2}^{2}(x) \leq \frac{1}{1-x} \quad \forall x<1, \\
& \xi_{1}\left[v_{1}^{2}(0)+v_{1}(0) v_{1}\left(\xi_{1}\right)+v_{1}^{2}\left(\xi_{1}\right)\right] \leq 3 \\
& \left(1-\xi_{2}\right)\left[v_{2}^{2}(1)+v_{2}(1) v_{2}\left(\xi_{2}\right)+v_{2}^{2}\left(\xi_{2}\right)\right] \leq 3 \\
& \left|v_{1}^{\prime}(x)\right|\left(x-x_{0}\right) \leq x_{0}^{-1 / 2} \quad \forall x_{0} \geq \xi_{1}, x \in\left[x_{0}, 1\right], \\
& \left|v_{2}^{\prime}(x)\right|\left(x_{0}-x\right) \leq\left(1-x_{0}\right)^{-1 / 2} \quad \forall x_{0} \leq \xi_{2}, x \in\left[0, x_{0}\right] .
\end{aligned}
$$

Proof. Estimates (19) follow by the $L^{2}$ constraint:

$$
1 \geq \int_{0}^{x} v_{1}^{2} \geq x v_{1}^{2}(x), \quad 1 \geq \int_{x}^{1} v_{2}^{2} \geq(1-x) v_{2}^{2}(x) .
$$

For the other estimates, it is crucial to observe that $\lambda_{1}-g_{1}\left(v_{2}^{2}\left(\xi_{1}\right)\right)=0$, as $v_{1}^{\prime \prime}\left(\xi_{1}\right)=0\left(\xi_{1}\right.$ is a point in $(0,1)$ where $v_{1}^{\prime}$ achieves its minimum). The function $\lambda_{1}-g_{1}\left(v_{2}^{2}\right)$ is decreasing, so by the equation for $v_{1}$ in (1) we deduce that $v_{1}$ is concave on $\left[0, \xi_{1}\right]$ and convex on $\left[\xi_{1}, 1\right]$.

Concavity implies that

$$
v_{1}(x) \geq v_{1}(0)+\xi_{1}^{-1}\left(v_{1}\left(\xi_{1}\right)-v_{1}(0)\right) x
$$

in $\left[0, \xi_{1}\right]$. By invoking the $L^{2}$ constraint of $v_{1}$ and integrating,

$$
1 \geq \int_{0}^{\xi_{1}} v_{1}^{2}(x) d x \geq \frac{\xi_{1}}{3}\left[v_{1}^{2}(0)+v_{1}(0) v_{1}\left(\xi_{1}\right)+v_{1}^{2}\left(\xi_{1}\right)\right],
$$

and (20) follows. Similarly, concavity of $v_{2}$ on $\left[\xi_{2}, 1\right]$ produces (21).

By convexity of $v_{1}$ on $\left[x_{0}, 1\right]$, and (19),

$$
-v_{1}^{\prime}(x)\left(x-x_{0}\right) \leq-v_{1}^{\prime}(x)\left(x-x_{0}\right)+v_{1}(x) \leq v_{1}\left(x_{0}\right) \leq x_{0}^{-1 / 2},
$$

for all $x \in\left[x_{0}, 1\right]$, and we have (22). Similarly, (23) follows by concavity of $v_{2}$ on $\left[0, x_{0}\right]$.

Lemma 7.2. Suppose that $\left\|v_{1, n}\right\|_{\infty} \leq C_{1}$. Then,

$$
v_{1, n}^{2}(x) \geq \frac{1}{2} \quad \text { in }\left[0, a_{1}\right]
$$

where $a_{1}=a_{1}\left(C_{1}\right)$. Similarly, if $\left\|v_{2, n}\right\|_{\infty} \leq C_{2}$,

$$
v_{2, n}^{2}(x) \geq \frac{1}{2} \quad \text { in }\left[a_{2}, 1\right]
$$

where $a_{2}=a_{2}\left(C_{2}\right)$.

Proof. In view of the $L^{2}$ constraint on $v_{1}$ and its monotonicity we have that

$$
1=\int_{0}^{x} v_{1}^{2}+\int_{x}^{1} v_{1}^{2} \leq x v_{1}^{2}(0)+(1-x) v_{1}^{2}(x)
$$

for all $x \in[0,1]$. Therefore, if $a_{1}=\left(2 C_{1}^{2}-1\right)^{-1}$,

$$
v_{1}^{2}\left(a_{1}\right) \geq \frac{1-a_{1} v_{1}^{2}(0)}{1-a_{1}} \geq \frac{1-a_{1} C_{1}^{2}}{1-a_{1}}=\frac{1}{2} .
$$

The assertion for $v_{1}$ follows. The estimate (25) for $v_{2}$ is analogous. 
Lemma 7.3. For both $i$ it holds

$$
\begin{gathered}
\left|v_{i, n}^{2}(0)-v_{i, n}^{2}(1)\right| \leq 2 \frac{\lambda_{i, n}}{\nu}, \\
\nu\left\|v_{i, n}^{\prime}\right\|_{\infty}^{2} \leq \lambda_{i, n}\left\|v_{i, n}\right\|_{\infty}^{2} .
\end{gathered}
$$

Proof. We will prove the assertion when $i=1$, the argument is analogous when $i=2$. Multiplying the equation for $v_{1}$ by $v_{1}$ and integrating on $[0, x]$ yields

$$
-\nu v_{1}^{\prime}(x) v_{1}(x)+\nu \int_{0}^{x}\left(v_{1}^{\prime}\right)^{2}=\int_{0}^{x}\left(\lambda_{1}-g_{1}\right) v_{1}^{2},
$$

thus

$$
-\frac{\nu}{2}\left(v_{1}^{2}\right)^{\prime}(x)=-\nu v_{1}^{\prime}(x) v_{1}(x) \leq \lambda_{1}
$$

By integrating again on $[0,1]$ we obtain (26).

On the other hand, testing the equation for $v_{1}$ by $v_{1}^{\prime}$ and integrating on $\left[0, \xi_{1}\right]$ we obtain

$$
\frac{\nu}{2} v_{1}^{\prime}\left(\xi_{1}\right)^{2}=\frac{\lambda_{1}}{2} v_{1}(0)^{2}+\int_{0}^{\xi_{1}} g_{1} v_{1} v_{1}^{\prime}
$$

and (27) follows since $v_{1}^{\prime} \leq 0$ in $[0,1]$.

After the above preliminary estimates, the first part of our analysis is devoted to show that $\mathcal{C}_{1}$ enjoys uniform $L^{\infty}$ bounds as $\nu \rightarrow 0$. To this aim we need two preliminary lemmas.

Lemma 7.4. Suppose that, for some $i,\left\|v_{i, n}\right\|_{\infty} \leq C$ and $\lambda_{j, n} \rightarrow 0$. Then, there exists $C^{\prime}>0$ that does not depend on $n$ such that

$$
\lambda_{i, n} \leq C^{\prime} \nu_{n}
$$

Proof. We will detail the proof in the case $i=1$. Note that

$$
g_{2}\left(v_{1}^{2}(x)\right)-\lambda_{2} \geq g_{2}(1 / 2)-\lambda_{2} \geq C_{g}^{-1} / 2-\lambda_{2} \geq C_{g}^{-1} / 4 \quad \text { in }\left[0, a_{1}\right]
$$

by the monotonicity of $g_{2},(24)$, (2) and $\lambda_{2} \rightarrow 0$. Hence,

$$
-v_{2}^{\prime \prime}=-\frac{g_{2}\left(v_{1}^{2}\right)-\lambda_{2}}{\nu} v_{2} \leq \frac{C_{g}^{-1}}{4 \nu} v_{2}
$$

in $\left(0, a_{1}\right)$, and Lemma 6.5 (or better Remark 6.6) allows to conclude that

$$
v_{2}(x) \leq 2 v_{2}\left(a_{1}\right) e^{-C / \sqrt{\nu}} \text { in }\left[0, a_{1} / 2\right],
$$

for some $C=C\left(a_{1}, C_{g}^{-1}\right)>0$.

Recalling Definition [1.1, we choose $w(x):=\sqrt{\frac{4}{a_{1}}} \cos \left(\frac{\pi}{a_{1}} x\right)$ for $x \in\left[0, a_{1} / 2\right]$ and $w \equiv 0$ in $\left[a_{1} / 2,1\right]$ to conclude that, for some $C^{\prime}>0$,

$$
\lambda_{1} \leq \int_{0}^{a_{1} / 2} \nu\left(w^{\prime}\right)^{2}+g_{1}\left(v_{2}^{2}\right) w^{2} \leq \frac{\nu \pi}{a_{1}}+g_{1}\left(4 v_{2}^{2}\left(a_{1}\right) e^{-2 C / \sqrt{\nu}}\right) \leq \frac{\nu \pi}{a_{1}}+\frac{4 C_{g} e^{-2 C / \sqrt{\nu}}}{1-a_{1}} \leq C^{\prime} \nu,
$$

by (21), (28) and (19). 
Lemma 7.5. Suppose that, for some $i,\left\|v_{i, n}\right\|_{\infty} \rightarrow+\infty$. Then,

$$
\nu\left\|v_{i, n}^{\prime}\right\|_{\infty}^{2} \leq C \lambda_{j, n}
$$

for some $C>0$ that does not depend on $n$.

Proof. We will detail the proof in the case $i=1$, thus assuming

$$
v_{1}(0) \rightarrow+\infty
$$

Note that $\left|v_{2}^{\prime}\right| \leq c_{2}$ in $[0,1 / 2]$ for some $c_{2}>0$. Indeed, if $v_{2}$ is bounded then Lemma 6.7 and Lemma 7.4 imply that $\lambda_{2} \leq C_{2}^{\prime} \nu$ for some $C_{2}^{\prime}>0$, and by (27) it follows that $\left\|v_{2}^{\prime}\right\|_{\infty}^{2} \leq C_{2}^{\prime}\left\|v_{2}\right\|_{\infty}^{2}$. On the other hand, if $v_{2}(1)$ is unbounded, then $\xi_{2} \rightarrow 1$ (see (21)), and then we have the required bound by (23) (choose, for example, $x_{0}=3 / 4$ ).

We now integrate the equation for $v_{2}$ on $\left[\xi_{1}, 1 / 2\right]$, use (2) and $\int v_{2}^{2}=1$ to obtain

$$
C_{g}^{-1} \int_{\xi_{1}}^{1 / 2} v_{1}^{2} v_{2} \leq \int_{\xi_{1}}^{1 / 2} g_{2}\left(v_{1}^{2}\right) v_{2}=\lambda_{2} \int_{\xi_{1}}^{1 / 2} v_{2}+\nu\left(v_{2}^{\prime}(1 / 2)-v_{2}^{\prime}\left(\xi_{1}\right)\right) \leq \lambda_{2}+2 c_{2} \nu
$$

Let $\bar{T}_{1}$ be the function

$$
\bar{T}_{1}(x)=\nu\left(v_{1}^{\prime}(x)\right)^{2}+\left[\lambda_{1}-g_{1}\left(v_{2}^{2}(x)\right)\right] v_{1}^{2}(x)+2 \int_{1 / 2}^{x} g_{1}^{\prime}\left(v_{2}^{2}(\sigma)\right) v_{2}^{\prime}(\sigma) v_{2}(\sigma) v_{1}^{2}(\sigma) d \sigma .
$$

$\bar{T}_{1}$ is easily verified to be constant in $[0,1]$. Since $\lambda_{1}-g_{1}\left(v_{2}^{2}(x)\right)$ is decreasing and $\lambda_{1}-g_{1}\left(v_{2}^{2}\left(\xi_{1}\right)\right)=$ $0, \lambda_{1}-g_{1}\left(v_{2}^{2}(1 / 2)\right) \leq 0$, as $\xi_{1} \leq 1 / 2\left(\xi_{1} \rightarrow 0\right.$ because $\left.v_{1}(0) \rightarrow+\infty\right)$. Hence,

$$
\begin{aligned}
\nu\left(v_{1}^{\prime}\left(\xi_{1}\right)\right)^{2}+2 \int_{1 / 2}^{\xi_{1}} g_{1}^{\prime}\left(v_{2}^{2}\right) v_{2}^{\prime} v_{2} v_{1}^{2} d \sigma= & \bar{T}_{1}\left(\xi_{1}\right) \\
& =\bar{T}_{1}(1 / 2)=\nu\left(v_{1}^{\prime}(1 / 2)\right)^{2}+\left[\lambda_{1}-g_{1}\left(v_{2}^{2}(1 / 2)\right)\right] v_{1}^{2}(1 / 2)
\end{aligned}
$$

and

$$
\nu\left\|v_{1}^{\prime}\right\|_{\infty}^{2}=\nu\left(v_{1}^{\prime}\left(\xi_{1}\right)\right)^{2} \leq \nu\left(v_{1}^{\prime}(1 / 2)\right)^{2}+2 \int_{\xi_{1}}^{1 / 2} g_{1}^{\prime}\left(v_{2}^{2}\right) v_{2}^{\prime} v_{1}^{2} v_{2} d \sigma \leq C\left(\nu+\lambda_{2}\right)
$$

The last bound comes from $\left|v_{2}^{\prime}\right| \leq c_{2},\left|v_{2}\right| \leq 1+c_{2} / 2$, (29) and $\left|v_{1}^{\prime}\right| \leq c_{1}$ in $[1 / 2,1]$ (use (22): $v_{1}$ is unbounded and $\xi_{1} \rightarrow 0$ ).

As already mentioned, the previous results allow to obtain uniform bounds for the sequence we are considering.

Lemma 7.6. There exists $C_{\infty}>0$, that does not depend on $n$, such that

$$
\left\|v_{i, n}\right\|_{\infty} \leq C_{\infty}, \quad i=1,2
$$

Proof. Without loss of generality, we can assume by contradiction that

$$
v_{1}(0) \rightarrow \infty, \quad \text { and } \lambda_{2} \leq \lambda_{1} .
$$

Indeed, if both $v_{1}(0)$ and $v_{2}(1)$ are unbounded, such condition can be guaranteed by interchanging the role of $v_{1}$ and $v_{2}$. Otherwise, suppose that, say, $v_{1}(0) \rightarrow \infty$ and $v_{2}$ is bounded: by Lemmas 6.7 and 7.4 there exists $C>0$ such that $\lambda_{2} \leq C \nu$, while $\lambda_{1} / \nu \rightarrow \infty$ (otherwise $v_{1}$ would be 
bounded in view of (26)). Therefore, $\lambda_{2} \leq \lambda_{1}$ whenever $\nu$ is sufficiently small and we infer, by Lemma 7.5, the existence of $C>0$ such that

$$
\left\|v_{1}^{\prime}\right\|_{\infty} \leq C \sqrt{\frac{\lambda_{1}}{\nu}}
$$

We proceed as in the proof of Lemma 6.7, by defining the blow-up sequences

$$
\tilde{v}_{1}(x):=\frac{1}{v_{1}(0)} v_{1}\left(x \sqrt{\frac{\nu}{\lambda_{1}}}\right), \quad \tilde{v}_{2}(x):=v_{2}\left(x \sqrt{\frac{\nu}{\lambda_{1}}}\right),
$$

Note that $0 \leq \tilde{v}_{1} \leq 1$ in $\left[0, \lambda_{1}^{1 / 2} \nu^{-1 / 2}\right]$, and that $\tilde{v}_{i}(0)=1$. Since, in such interval,

$$
\left|\tilde{v}_{1}^{\prime}(x)\right|=\frac{1}{v_{1}(0)} \sqrt{\frac{\nu}{\lambda_{1}}}\left|v_{1}^{\prime}\left(x \sqrt{\frac{\nu}{\lambda_{1}}}\right)\right| \leq \frac{C}{v_{1}(0)} \rightarrow 0
$$

(we used (30)), we deduce that $\tilde{v}_{1} \rightarrow V \equiv 1$, uniformly in every $[a, b] \subset[0,+\infty$ ). As a consequence, in any such interval,

$$
-\tilde{v}_{2}^{\prime \prime}=\left(\frac{\lambda_{2}}{\lambda_{1}}-\frac{g_{2}\left(v_{1}(0) \tilde{v}_{1}^{2}\right)}{\lambda_{1}}\right) \tilde{v}_{2} \leq\left(1-\frac{C_{g}^{-1}}{2 \lambda_{1}} v_{1}^{2}(0)\right) \tilde{v}_{2} \leq-C^{2} \frac{v_{1}^{2}(0)}{\lambda_{1}} \tilde{v}_{2}
$$

with $C>0$, and Remark 6.6 applies, yielding

$$
\tilde{v}_{2}(x) \leq \tilde{v}_{2}(b+1) e^{-C v_{1}(0) / \sqrt{\lambda_{1}}} \leq 2 e^{-C v_{1}(0) / \sqrt{\lambda_{1}}} \quad \text { for } x \in[0, b],
$$

for $\nu$ sufficiently small (recall (19) $)$. Then

$$
\frac{g_{1}\left(\tilde{v}_{2}^{2}\right)}{\lambda_{1}} \leq \frac{C_{1} e^{-C_{2} v_{1}(0) / \sqrt{\lambda_{1}}}}{\lambda_{1}} \leq \frac{C_{3}}{v_{1}^{2}(0)} \rightarrow 0
$$

and we can plug such estimate in the equation for $\tilde{v}_{1}$

$$
-\tilde{v}_{1}^{\prime \prime}=\left(1-\frac{g_{1}\left(\tilde{v}_{2}^{2}\right)}{\lambda_{1}}\right) \tilde{v}_{1}
$$

in order to pass to the limit and obtain

$$
-V^{\prime \prime}=V \quad \text { in }(0,+\infty),
$$

in contradiction with the fact that $V \equiv 1$.

Uniform $L^{\infty}$ bounds readily provide Lipschitz ones, thus yielding convergence to some limiting profiles.

Proposition 7.7. There exists $C_{\infty}^{\prime}>0$, not depending on $n$, such that

$$
\left\|v_{i, n}^{\prime}\right\|_{\infty} \leq C_{\infty}^{\prime} \quad i=1,2
$$

As a consequence, up to subsequences,

$$
v_{i, n} \rightarrow V_{i} \text { in } C^{0, \alpha}([0,1]), \quad \text { with } \int_{0}^{1} V_{1}^{2}=\int_{0}^{1} V_{2}^{2}=1 \text { and } V_{1} \cdot V_{2} \equiv 0 \text { in }[0,1],
$$

and

as $n \rightarrow+\infty$.

$$
\frac{\lambda_{i, n}}{\nu_{n}} \rightarrow \ell_{i}>0,
$$


Proof. Lemma 7.6 guarantees the uniform $L^{\infty}$ bound for $v_{1}, v_{2}$, hence $\lambda_{1}, \lambda_{2} \rightarrow 0$ by Lemma 6.3, As a consequence we can apply Lemma 7.4 for both $i$, obtaining that there exists $C_{i}^{\prime}>0$ that does not depend on $\nu$ such that

$$
\lambda_{i} \leq C_{i}^{\prime} \nu
$$

This implies that both $\nu\left\|v_{i}^{\prime}\right\|_{\infty}^{2} \leq \nu C_{i}^{\prime}\left\|v_{1}\right\|_{\infty}^{2}$, by (27), and, up to subsequences, both $v_{i} \rightarrow V_{i}$ in $C^{0 . \alpha}$ and $\lambda_{i} / \nu \rightarrow \ell_{i} \geq 0$. Since uniform convergence implies $L^{2}$-one, the required properties for the limiting profiles $V_{i}$ follow (recall Corollary 2.5), and the only thing that remains to be proved is that both $\ell_{i}>0$.

Assume by contradiction that, for instance, $\ell_{1}=0$. Then we can use equation (26) to infer that $V_{1} \equiv 1$, in contradiction with (31).

Remark 7.8. Once we know that $v_{i, n} \rightarrow V_{i}$ uniformly, the strong $H^{1}$ convergence follows by standard arguments. Indeed, integrating the equations we have

$$
0 \leq \frac{1}{\nu} \int_{0}^{1} g_{i}\left(v_{j, n}^{2}\right) v_{i, n} d x=\frac{\lambda_{i, n}}{\nu} \int_{0}^{1} v_{i, n} d x \leq C
$$

therefore, testing with $v_{i, n}-V_{i}$ we infer

$$
\int_{0}^{1} v_{i, n}^{\prime}\left(v_{i, n}^{\prime}-V_{i}^{\prime}\right) d x \leq \max _{[0,1]}\left|v_{i, n}-V_{i}\right| \cdot \frac{1}{\nu} \int_{0}^{1}\left(\lambda_{i, n}+g_{i}\left(v_{j, n}^{2}\right)\right) v_{i, n} d x \rightarrow 0 .
$$

As a consequence, weak $H^{1}$ convergence implies convergence in norm, and finally strong $H^{1}$ one.

The remaining part of the section will be devoted to fully characterize the limits $V_{i}, \ell_{i}$. To this aim, we need a sharper analysis of the convergence of $v_{i, n}$.

Lemma 7.9. Suppose that, as $n \rightarrow+\infty, v_{1, n}\left(y_{n}\right) \geq c \nu_{n}^{1 / 2-\epsilon}$ for some $y_{n} \in[0,1), c>0$, $0<\epsilon \leq 1 / 2$. Then there exists $c_{1}>0$ such that

$$
v_{2, n}(x) \leq 2 v_{2, n}\left(y_{n}\right) e^{-c_{1}\left(y_{n}-x\right) \nu_{n}^{-\epsilon}} \quad \text { in }\left[0, y_{n}\right] .
$$

Proof. By the monotonicity of $v_{1}$, (2) and (32),

$$
g_{2}\left(v_{1}^{2}(x)\right)-\lambda_{2} \geq C_{g}^{-1} v_{1}^{2}(x)-\lambda_{2} \geq \frac{C_{g}^{-1} c^{2}}{2} \nu^{1-2 \epsilon} \quad \text { in }[0, y]
$$

as $\nu \rightarrow 0$. Hence,

$$
-v_{2}^{\prime \prime}=-\frac{g_{2}\left(v_{1}^{2}\right)-\lambda_{2}}{\nu} v_{2} \leq-\frac{C_{g}^{-1} c^{2}}{2} \nu^{-2 \epsilon} v_{2}
$$

in $(0, y)$, and we can conclude using Remark 6.6

Remark 7.10. A direct consequence of the previous lemma, which will be used thoroughly in the sequel, is that if $\liminf _{\nu \rightarrow 0} v_{1}(y)>0$ for some $y \in[0,1)$, then there exists $c_{2}>0, y<b<1$ (that does not depend on $\nu$ ) such that

$$
v_{2}(x) \leq C_{\infty} e^{-\frac{c_{2}}{\sqrt{\nu}}} \text { in }[0, b] .
$$

Indeed, the assumption guarantees that $v_{1}(y) \geq 2 c>0$ for some $c>0$, so, by Proposition 7.7, $v_{1}\left(y^{\prime}\right) \geq c$ for some $y^{\prime}>y$. Hence,

$$
v_{2}(x) \leq C_{\infty} e^{-c_{1}\left(y^{\prime}-x\right) \nu^{-1 / 2}} \quad \text { in }\left[0, y^{\prime}\right]
$$


that implies (33) if we choose $y<b<y^{\prime}$, and $c_{2}=c_{2}\left(c_{1}, b, y, y^{\prime}\right)>0$.

Note that $v_{1}(0) \geq 1$ for all $\nu$ (otherwise the mass constraint $\int_{0}^{1} v_{1}^{2} d x=1$ would be violated), thus

$$
v_{2}(0) \leq C_{\infty} e^{-c_{2} \nu^{-1 / 2}}=o\left(\nu^{a}\right) \text { for all } a>0 .
$$

for some $c_{2}>0$.

Analogous conclusions hold if $v_{1}$ and $v_{2}$ are interchanged.

Lemma 7.11. The limit $V_{i}, \ell_{i}$ satisfy, in $[0,1]$,

$$
\begin{aligned}
& V_{1}(x)=\frac{2}{\sqrt{\pi}} \sqrt[4]{\ell_{1}} \cos \left(\sqrt{\ell_{1}} x\right) \cdot \chi_{\left[0, \frac{\pi}{2 \sqrt{\ell_{1}}}\right]}(x) \\
& V_{2}(x)=\frac{2}{\sqrt{\pi}} \sqrt[4]{\ell_{2}} \cos \left(\sqrt{\ell_{2}}(x-1)\right) \cdot \chi_{\left[1-\frac{\pi}{2 \sqrt{\ell_{2}}}, 1\right]}(x) .
\end{aligned}
$$

Moreover, as $n \rightarrow+\infty$,

$$
\xi_{1, n} \rightarrow \frac{\pi}{2 \sqrt{\ell_{1}}}, \quad \xi_{2, n} \rightarrow 1-\frac{\pi}{2 \sqrt{\ell_{2}}}
$$

Proof. Let $x_{1}>0$ be such that $\left[0, x_{1}\right)=\left\{x: V_{1}(x)>0\right\}\left(V_{1}\right.$ is identically zero in $\left.\left[x_{1}, 1\right]\right)$. If $y<x_{1}, v_{1}(y)$ is bounded away from zero, uniformly with respect to $\nu$, hence $v_{2}(x) \leq C_{\infty} e^{-\frac{c_{2}}{\sqrt{\nu}}}$ in $[0, y]$ by (33). Therefore, $g_{1}\left(v_{2}^{2}\right)=o(\nu)$ uniformly in $[0, y]$, that is

$$
\frac{\lambda_{1}-g_{1}\left(v_{2}^{2}\right)}{\nu}=o(1)
$$

uniformly on compact subsets of $\left[0, x_{1}\right)$. Hence, we might pass to the limit (weakly) into the equation for $v_{1}$ : let $\varphi$ be a smooth test function, with support laying in $\left[0, x_{1}\right)$. The equation reads

$$
\int_{0}^{x_{1}} v_{1}^{\prime} \varphi^{\prime} d x=\int_{0}^{x_{1}} \frac{\lambda_{1}-g_{1}\left(v_{2}^{2}(x)\right)}{\nu} v_{1} \varphi d x
$$

and passing to the limit (Proposition 7.7 ensures weak convergence in $H^{1}((0,1))$ of $v_{i}$ to $V_{i}$ ),

$$
-V_{1}^{\prime \prime}=\ell_{1} V_{1} \text { in }\left(0, x_{1}\right),
$$

$V_{1}^{\prime}(0)=0$ and $V_{1}\left(x_{1}\right)=0$. Thus, being $V_{1}$ positive, it has to be of the form $A \cos \left(\sqrt{\ell_{1}} x\right)$ in $\left(0, x_{1}\right)$, for some $A>0$. This forces $x_{1}=\pi /\left(2 \sqrt{\ell_{1}}\right)$. Moreover, $\int_{0}^{1} V_{1}^{2}=1$, since by uniform convergence the $L^{2}$-constraint passes to the limit, and $A$ must satisfy $A=\frac{2}{\sqrt{\pi}} \sqrt[4]{\ell_{1}}$. The characterization of $V_{2}$ is analogous.

As for the second assertion, we argue that $v_{1}\left(\xi_{1}\right) \rightarrow 0$. If not, $v_{2}\left(\xi_{1}\right) \leq C_{\infty} e^{-\frac{c_{2}}{\sqrt{\nu}}}=o\left(\nu^{1 / 2}\right)$ by (33), that is not compatible with $g_{1}\left(v_{2}^{2}\left(\xi_{1}\right)\right)=\lambda_{1} \geq c_{1} \nu$. Hence, $\lim \xi_{1} \geq x_{1}$. Suppose that $\lim \xi_{1}>x_{1}$; note that $v_{1}$ is concave on $\left(0, \xi_{1}\right)$, so $v_{1}(x) \geq v_{1}(0)+\left(v_{1}\left(\xi_{1}\right)-v_{1}(0)\right) x / \xi_{1}$ in $\left[0, \xi_{1}\right]$. We infer

$$
\lim v_{1}\left(x_{1}\right) \geq v_{1}(0)\left(1-\lim \frac{x_{1}}{\xi_{1}}\right)>0
$$

which contradicts $v_{1}\left(x_{1}\right) \rightarrow V_{1}\left(x_{1}\right)=0$. Then, $\xi_{1} \rightarrow x_{1}=\pi /\left(2 \sqrt{\ell_{1}}\right)$.

Lemma 7.12. For all $a>2$ it holds true that

$$
\int_{0}^{1} v_{i, n}^{a} v_{j, n}^{2} d x=o\left(\nu_{n}\right), \quad \text { as } n \rightarrow+\infty .
$$


Proof. The assertion will be proved for $i=1$, the other case being completely analogous. Let us define $y \in(0,1)$ as the unique point such that

$$
v_{1}(y)=\nu^{1 / 6}
$$

The point $y$ is well defined and bounded away from zero because $v_{1}$ is uniformly strictly positive in a neighborhood of $x=0$ and $v_{1}(1)=o(\nu)$ (by (34) for $v_{1}$ ). By Lemma 7.9 we also have $v_{2}(x) \leq C e^{-c_{1}(y-x) \nu^{-1 / 3}}$ in $[0, y]$. We split the interval $[0,1]$ into two subintervals, and exploit $\left\|v_{i}\right\|_{\infty} \leq C_{\infty}$

In $\left[0, y-\nu^{1 / 6}\right]$, by monotonicity we have $v_{2}(x) \leq C e^{-c_{1} \nu^{-1 / 6}}$ and

$$
\int_{0}^{y-\nu^{1 / 6}} v_{1}^{a} v_{2}^{2} d x \leq C e^{-2 c_{1} \nu^{-1 / 6}}=o(\nu) .
$$

In $\left[y-\nu^{1 / 6}, 1\right]$, recalling that $\int_{0}^{1} v_{1}^{2} v_{2}^{2} \leq C_{g}^{-1} \lambda_{1} \leq C \nu$ by (2) and (32), we obtain

$$
\int_{y-\nu^{1 / 6}}^{1} v_{1}^{a} v_{2}^{2} d x \leq v_{1}^{a-2}\left(y-\nu^{1 / 6}\right) \cdot \int_{0}^{1} v_{1}^{2} v_{2}^{2} \leq v_{1}^{a-2}\left(y-\nu^{1 / 6}\right) \cdot C \nu=o(\nu),
$$

as $v_{1}\left(y-\nu^{1 / 6}\right)$ goes to zero (recall that $v_{1}$ converges uniformly, and that $v_{1}(y) \rightarrow 0$ ).

The last part of our analysis focuses on the "interface" between $v_{1}$ and $v_{2}$, namely we are going to consider the point $x_{m}=x_{m, n} \in(0,1)$ such that

$$
m_{n}=v_{1}\left(x_{m, n}\right)=v_{2}\left(x_{m, n}\right) .
$$

We follow ideas introduced in [5], to treat the one-dimensional variational case. Note that by strict monotonicity of $v_{i, n}, x_{m, n} \in(0,1)$ is well-defined, and

$$
m_{n} \rightarrow 0, \quad x_{m, n} \rightarrow x_{0} \in(0,1),
$$

in view of (31) and the fact that $v_{1, n}$ and $v_{2, n}$ are bounded away from zero in neighborhoods of $x=0$ and $x=1$ respectively (see Lemma 7.2 ).

In what follows, we will write

$$
g_{i}(s)=\gamma_{i} s+h_{i}(s), \quad \text { for all } s \geq 0,
$$

where $\gamma_{i}=g_{i}^{\prime}(0)>0, h_{i}(0)=0, h_{i}^{\prime}(0)=0$.

Remark 7.13. The functions $h_{i}$ have to be considered as "lower order terms" in the vanishing viscosity limit, and we will usually Taylor expand them around $s=0$, namely

$$
h_{i}\left(v_{j}^{2}(x)\right)=a_{i}(x) v_{j}^{4}(x), \quad h_{i}^{\prime}\left(v_{j}^{2}\right)=b_{i}(x) v_{j}^{2}(x),
$$

where $\left|a_{i}(x)\right|,\left|b_{i}(x)\right| \leq C$ for some universal constant $C>0$ (depending on $g^{\prime \prime}$ ).

The "joint energy" is going to be crucial in our analysis:

$$
\begin{array}{r}
T(x):=\frac{1}{\gamma_{1}}\left[\nu\left(v_{1}^{\prime}(x)\right)^{2}+\left[\lambda_{1}-h_{1}\left(v_{2}^{2}(x)\right)\right] v_{1}^{2}(x)+2 \int_{x_{m}}^{x} h_{1}^{\prime}\left(v_{2}^{2}(\sigma)\right) v_{2}^{\prime}(\sigma) v_{2}(\sigma) v_{1}^{2}(\sigma) d \sigma\right] \\
+\frac{1}{\gamma_{2}}\left[\nu\left(v_{2}^{\prime}(x)\right)^{2}+\left[\lambda_{2}-h_{2}\left(v_{1}^{2}(x)\right)\right] v_{2}^{2}(x)-2 \int_{x}^{x_{m}} h_{2}^{\prime}\left(v_{1}^{2}(\sigma)\right) v_{1}^{\prime}(\sigma) v_{1}(\sigma) v_{2}^{2}(\sigma) d \sigma\right] \\
-v_{1}^{2}(x) v_{2}^{2}(x) .
\end{array}
$$

Of course, along any pair $\left(v_{1, n}, v_{2, n}\right), T_{n}(x)=T(x)$ is constant (indeed $\left.T_{n}^{\prime}(x) \equiv 0\right)$. 
Lemma 7.14. It holds

$$
\frac{\lambda_{1, n}}{\gamma_{1}} v_{1, n}^{2}(0)+o\left(\nu_{n}\right)=T_{n}=\frac{\lambda_{2, n}}{\gamma_{2}} v_{2, n}^{2}(1)+o\left(\nu_{n}\right),
$$

as $n \rightarrow+\infty$.

Proof. Note firstly that

$$
\left|\int_{x_{m}}^{x} h_{i}^{\prime}\left(v_{j}^{2}\right) v_{j}^{\prime} v_{j} v_{i}^{2} d \sigma\right| \leq \int_{0}^{1}\left|b_{i} v_{j}^{\prime}\right| v_{j}^{3} v_{i}^{2} d \sigma \leq C \int_{0}^{1} v_{j}^{3} v_{i}^{2}=o(\nu),
$$

by Lemma 7.12 and Remark 7.13. Note also that $v_{2}^{2}(0)=o(\nu)$ by (34).

Therefore, being $v_{1}$ bounded by $C_{\infty}$,

$$
\begin{aligned}
T(0)= & \frac{\lambda_{1}}{\gamma_{1}} v_{1}^{2}(0)-\frac{v_{1}^{2}(0)}{\gamma_{1}} h_{1}\left(v_{2}^{2}(0)\right)+\frac{2}{\gamma_{1}} \int_{x_{m}}^{0} h_{1}^{\prime}\left(v_{2}^{2}\right) v_{2}^{\prime} v_{2} v_{1}^{2} d \sigma \\
& +\frac{\lambda_{2}-h_{2}\left(v_{1}^{2}(0)\right)}{\gamma_{2}} v_{2}^{2}(0)-\frac{2}{\gamma_{2}} \int_{0}^{x_{m}} h_{2}^{\prime}\left(v_{1}^{2}\right) v_{1}^{\prime} v_{1} v_{2}^{2} d \sigma-v_{1}^{2}(0) v_{2}^{2}(0)=\frac{\lambda_{1}}{\gamma_{1}} v_{1}^{2}(0)+o(\nu) .
\end{aligned}
$$

Similarly,

$$
T(1)=\frac{\lambda_{2}}{\gamma_{2}} v_{2}^{2}(1)+o(\nu)
$$

Lemma 7.15. It holds true that

$$
\limsup _{n \rightarrow+\infty} \frac{m_{n}^{4}}{\nu_{n}}<+\infty
$$

Proof. Arguing by contradiction,

$$
\frac{m^{4}}{\nu} \rightarrow \infty
$$

possibly along a subsequence. Let $\tilde{v}_{i}(x):=\frac{1}{m} v_{i}\left(x_{m}+x \frac{\sqrt{\nu}}{m}\right)$. Then, $\tilde{v}_{i}$ solves

$$
-\tilde{v}_{i}^{\prime \prime}=\left(\frac{\lambda_{i}}{m^{2}}-\gamma_{i} \tilde{v}_{j}^{2}-\frac{h_{i}\left(m^{2} \tilde{v}_{j}^{2}\right)}{m^{2}}\right) \tilde{v}_{i}, \quad \text { in } I_{\nu}=\left(-\frac{m^{2}}{\nu} x_{m},\left(1-x_{m}\right) \frac{m^{2}}{\nu}\right) .
$$

Note that $I_{\nu}$ tends to the whole real line as $\nu \rightarrow 0\left(x_{m}\right.$ is bounded away from $x=0$ and $x=1$, and $\left.m^{2} \nu^{-1} \rightarrow \infty\right), \tilde{v}_{i}(0)=1$ and

$$
\left|\tilde{v}_{i}(y)-\tilde{v}_{i}(0)\right| \leq|y|\left\|v_{i}^{\prime}\right\|_{\infty} \frac{\sqrt{\nu}}{m^{2}} \rightarrow 0, \quad \text { for all } y \in[a, b] \subset I_{\nu} .
$$

Thus, $\tilde{v}_{i}$ converges uniformly on compact subsets of $I_{\nu}$, as $\left\|v_{i}^{\prime}\right\|_{\infty}$ is bounded by $C_{\infty}^{\prime}$. Moreover, $\lambda_{i} m^{-2} \rightarrow 0$ (by (32) ) and $h_{i}\left(m^{2} \tilde{v}_{j}^{2}\right) m^{-2} \rightarrow 0$ uniformly on compact subsets of $I_{\nu}$ (see Remark (7.13). Hence,

$$
\tilde{v}_{i} \rightarrow 1, \quad \tilde{v}_{i}^{\prime} \rightarrow 0 \quad \text { locally uniformly. }
$$

We then have

$$
\begin{gathered}
\sum_{i=1,2} \frac{1}{\gamma_{i}}\left[\left(\tilde{v}_{i}^{\prime}(0)\right)^{2}+\left(\frac{\lambda_{i}}{m^{2}}-\frac{h_{i}\left(m^{2} \tilde{v}_{j}^{2}(0)\right)}{m^{2}}\right) \tilde{v}_{i}^{2}(0)\right]-\tilde{v}_{1}^{2}(0) \tilde{v}_{2}^{2}(0)= \\
\frac{T\left(x_{m}\right)}{m^{4}}=\frac{\lambda_{1}}{\gamma_{1} m^{4}} v_{1}^{2}(0)+o\left(\nu / m^{4}\right) \geq \frac{c_{1} \nu}{\gamma_{1} m^{4}} v_{1}^{2}(0)+o\left(\nu / m^{4}\right) \geq 0
\end{gathered}
$$

by (38) and (32) when $\nu$ is close enough to zero. On the other hand, the left hand side of (40) goes to -1 as $\nu \rightarrow 0$ by (39), a contradiction. 
Lemma 7.16. As $n \rightarrow+\infty$, there exists $L>0$ such that

$$
\frac{m_{n}^{4}}{\nu_{n}} \rightarrow L
$$

Moreover,

$$
\liminf \left|v_{i, n}^{\prime}\left(x_{m, n}\right)\right|>0 .
$$

Proof. Let us assume by contradiction that

$$
\frac{m^{4}}{\nu} \rightarrow 0
$$

Let $\tilde{v}_{i}(x):=\frac{1}{m} v_{i}\left(x_{m}+m x\right)$. Then, $\tilde{v}_{i}$ solves

$$
-\tilde{v}_{i}^{\prime \prime}=\frac{m^{4}}{\nu}\left(\frac{\lambda_{i}}{m^{2}}-\gamma_{i} \tilde{v}_{j}^{2}-\frac{h_{i}\left(m^{2} \tilde{v}_{j}^{2}\right)}{m^{2}}\right) \tilde{v}_{i}, \quad \text { in } I_{\nu}=\left(-\frac{x_{m}}{m}, \frac{1-x_{m}}{m}\right) .
$$

Note that $I_{\nu}$ tends to the whole real line as $\nu \rightarrow 0, \tilde{v}_{i}(0)=1$ and $\left\|\tilde{v}_{i}^{\prime}\right\|_{\infty}=\left\|v_{i}^{\prime}\right\|_{\infty} \leq C_{\infty}^{\prime}$, so $\tilde{v}_{i}$ converges uniformly on compact subsets of $I_{\nu}$. Moreover, $\lambda_{i} m^{-2} \rightarrow 0$ and $h_{i}\left(m^{2} \tilde{v}_{j}^{2}\right) m^{-2} \rightarrow 0$ uniformly on compact subsets of $I_{\nu}$ (as in the proof of the Lemma 7.15). Hence, $\tilde{v}_{i} \rightarrow W_{i}$ locally in $C^{2}(\mathbb{R})$, and $W_{i}^{\prime \prime}=0$. Since $W_{i}$ is positive we have

$$
W_{i} \equiv 1 \quad \text { in } \mathbb{R}, i=1,2 .
$$

Therefore,

$$
\begin{aligned}
\sum_{i=1,2} \frac{1}{\gamma_{i}}\left[\left(\tilde{v}_{i}^{\prime}(0)\right)^{2}+\frac{m^{4}}{\nu}\left(\frac{\lambda_{i}}{m^{2}}-\frac{h_{i}\left(m^{2} \tilde{v}_{j}^{2}(0)\right)}{m^{2}}\right) \tilde{v}_{i}^{2}(0)\right]-\frac{m^{4}}{\nu} \tilde{v}_{1}^{2}(0) \tilde{v}_{2}^{2}(0) & = \\
\frac{T\left(x_{m}\right)}{\nu} & =\frac{\lambda_{1}}{\gamma_{1} \nu} v_{1}^{2}(0)+o(1) \geq \frac{c_{1}}{\gamma_{1}} v_{1}^{2}(0)+o(1)>0
\end{aligned}
$$

by (38) and (32) when $\nu \rightarrow 0$. However, the left hand side of (40) goes to zero as $\nu \rightarrow 0$ by (43), that is not possible. Hence, $L>0$.

To prove (42) we proceed as before, setting $\tilde{v}_{i}(x):=\frac{1}{m} v_{i}\left(x_{m}+m x\right)$. We have that $\tilde{v}_{i} \rightarrow W_{i}$ locally in $C^{2}(\mathbb{R})$, and $\left(W_{1}, W_{2}\right)$ solve

$$
\left\{\begin{array}{l}
W_{1}^{\prime \prime}=L \gamma_{1} W_{2}^{2} W_{1} \\
W_{2}^{\prime \prime}=L \gamma_{2} W_{1}^{2} W_{2}
\end{array}\right.
$$

in $\mathbb{R}$. $W_{1}$ and $W_{2}$ are also also positive and monotone, so $W_{i}^{\prime}(0) \neq 0$. We conclude by observing that $\left|v_{i}^{\prime}\left(x_{m}\right)\right|=\left|\tilde{v}_{i}^{\prime}(0)\right| \rightarrow\left|W_{i}^{\prime}(0)\right|>0$.

Lemma 7.17. As $n \rightarrow+\infty$, it holds true that

$$
\xi_{1, n} \leq x_{m, n} \leq \xi_{2, n},
$$

and

$$
\xi_{1, n} \rightarrow x_{0}, \quad \xi_{2, n} \rightarrow x_{0} .
$$


Proof. Since $\xi_{1}$ is the inflection point of $v_{1}$ we have

$$
C_{g}^{-1} v_{2}^{2}\left(\xi_{1}\right) \leq g_{1}\left(v_{2}^{2}\left(\xi_{1}\right)\right)=\lambda_{1} \leq C_{1}^{\prime} \nu,
$$

also by invoking (2) and (32). Hence, $v_{2}\left(\xi_{1}\right) \leq \sqrt{\left(C_{g} C_{1}^{\prime}\right) \nu}$, but $v_{2}\left(x_{m}\right)=m \sim \sqrt[4]{L \nu}$ by (41), so $v_{2}\left(\xi_{1}\right) \leq v_{2}\left(x_{m}\right)$ for $\nu$ sufficiently small. Monotonicity of $v_{2}$ implies that $\xi_{1} \leq x_{m}$, while $x_{m} \leq \xi_{2}$ is obtained by an analogous argument at the inflection point $\xi_{2}$ of $v_{2}$.

Suppose now that $\xi_{2}-\xi_{1}=4 \eta$ and $\eta$ is uniformly bounded away from zero as $\nu \rightarrow 0$. Assume, without loss of generality, that $x_{m} \in\left[\xi_{1}, \xi_{1}+2 \eta\right]$ (on the other hand, if $x_{m} \in\left[\xi_{1}+2 \eta, \xi_{2}\right]$ we interchange the roles of $v_{1}$ and $v_{2}$ ). Note that $\xi_{2}$ is the inflection point of $v_{2}$, so $v_{2}$ is convex on $\left(0, \xi_{2}\right)$, which provides $v_{2}(x) \geq v_{2}\left(x_{m}\right)+v_{2}^{\prime}\left(x_{m}\right)\left(x-x_{m}\right)$ for all $x \in\left(0, \xi_{2}\right)$. Therefore,

$$
v_{2}\left(\xi_{1}+3 \eta\right) \geq v_{2}\left(x_{m}\right)+v_{2}^{\prime}\left(x_{m}\right)\left(\xi_{1}+3 \eta-x_{m}\right) \geq c\left(\xi_{1}+3 \eta-x_{m}\right) \geq c \eta>0,
$$

for some positive $c$ in view of (42). Now we reason as in Remark 7.10 (in particular we apply (33) with $v_{1}$ and $v_{2}$ interchanged) to get

$$
v_{1}\left(\xi_{1}+3 \eta\right) \leq C_{\infty} e^{-\frac{c_{2}}{\sqrt{\nu}}}=o\left(\nu^{1 / 2}\right)
$$

but $C_{g}^{-1} v_{1}^{2}\left(\xi_{2}\right) \geq g_{2}\left(v_{1}^{2}\left(\xi_{2}\right)\right)=\lambda_{2} \geq c_{2} \nu$ (again by (22) and (32)), so $v_{1}\left(\xi_{2}\right) \geq v_{1}\left(\xi_{1}+3 \eta\right)$ as $\nu \rightarrow 0$. Being $v_{1}$ decreasing, $\xi_{2} \leq \xi_{1}+3 \eta=\xi_{2}-\eta$, which is impossible. Then, $0 \leq \xi_{2}-\xi_{1} \rightarrow 0$ follows, and the second assertion is proved as $x_{m} \rightarrow x_{0}$.

Proof of Theorem 1.8. In view of Propositions 3.3, 7.7 and Remark 7.8, the theorem will follow once we show that the following equalities hold:

$$
\frac{\ell_{2}}{\ell_{1}}=\left(\frac{\gamma_{2}}{\gamma_{1}}\right)^{2 / 3} \quad \text { and } \quad x_{0}=\frac{\sqrt[3]{\gamma_{2}}}{\sqrt[3]{\gamma_{1}}+\sqrt[3]{\gamma_{2}}}
$$

To this aim, we put together all the asymptotic information (as $\nu \rightarrow 0$ ) we obtained so far. Firstly, $\pi v_{1}^{2}(0) \sim 4 \sqrt{\ell_{1}}$ and $\pi v_{2}^{2}(1) \sim 4 \sqrt{\ell_{2}}$ by (35) and (36). Hence, if we divide (38) by $\nu$ we obtain

$$
\frac{\ell_{1} \sqrt{\ell_{1}}}{\gamma_{1}}=\frac{\ell_{2} \sqrt{\ell_{2}}}{\gamma_{2}}
$$

which is the first stated equality. Then, $x_{0}=\frac{\pi}{2 \sqrt{\ell_{1}}}$ by (37) and (44). Moreover,

$$
\frac{\pi}{2 \sqrt{\ell_{1}}}+\frac{\pi}{2 \sqrt{\ell_{2}}}=1
$$

By plugging (45) in the last equality we conclude.

\section{Funding}

Work partially supported by the PRIN-2012-74FYK7 Grant: "Variational and perturbative aspects of nonlinear differential problems", by the ERC Advanced Grant 2013 n. 339958: "Complex Patterns for Strongly Interacting Dynamical Systems - COMPAT", and by the INDAMGNAMPA grant "Analisi Globale, PDEs e Strutture Solitoniche" (2015). 


\section{References}

[1] Y. Achdou, M. Bardi, and M. Cirant. Mean field games models of segregation. preprint, 2015.

[2] A. Ambrosetti and G. Prodi. A primer of nonlinear analysis, volume 34 of Cambridge Studies in Advanced Mathematics. Cambridge University Press, Cambridge, 1993.

[3] T. Bartsch and L. Jeanjean. Normalized solutions for nonlinear Schrödinger systems. arxiv: 1507.04649, 072015.

[4] T. Bartsch, L. Jeanjean, and N. Soave. Normalized solutions for a system of coupled cubic Schrödinger equations on $\mathbb{R}^{3}$. arXiv:1506.02262, 062015.

[5] H. Berestycki, T.-C. Lin, J. Wei, and C. Zhao. On phase-separation models: asymptotics and qualitative properties. Arch. Ration. Mech. Anal., 208(1):163-200, 2013.

[6] H. Berestycki, S. Terracini, K. Wang, and J. Wei. On entire solutions of an elliptic system modeling phase separations. Adv. Math., 243:102-126, 2013.

[7] L. A. Caffarelli and F.-H. Lin. Singularly perturbed elliptic systems and multi-valued harmonic functions with free boundaries. J. Amer. Math. Soc., 21(3):847-862, 2008.

[8] L. A. Caffarelli and J.-M. Roquejoffre. Uniform Hölder estimates in a class of elliptic systems and applications to singular limits in models for diffusion flames. Arch. Ration. Mech. Anal., 183(3):457487, 2007.

[9] S.-M. Chang, C.-S. Lin, T.-C. Lin, and W.-W. Lin. Segregated nodal domains of two-dimensional multispecies Bose-Einstein condensates. Phys. D, 196(3-4):341-361, 2004.

[10] M. Cirant. Multi-population mean field games systems with Neumann boundary conditions. $J$. Math. Pures Appl. (9), 103(5):1294-1315, 2015.

[11] M. Conti, S. Terracini, and G. Verzini. Nehari's problem and competing species systems. Ann. Inst. H. Poincaré Anal. Non Linéaire, 19(6):871-888, 2002.

[12] M. Conti, S. Terracini, and G. Verzini. Asymptotic estimates for the spatial segregation of competitive systems. Adv. Math., 195(2):524-560, 2005.

[13] M. Conti, S. Terracini, and G. Verzini. Uniqueness and least energy property for solutions to strongly competing systems. Interfaces Free Bound., 8(4):437-446, 2006.

[14] M. G. Crandall and P. H. Rabinowitz. Bifurcation from simple eigenvalues. J. Functional Analysis, 8:321-340, 1971.

[15] E. N. Dancer, K. Wang, and Z. Zhang. Uniform Hölder estimate for singularly perturbed parabolic systems of Bose-Einstein condensates and competing species. J. Differential Equations, 251(10):2737-2769, 2011.

[16] E. Feleqi. The derivation of ergodic mean field game equations for several populations of players. Dyn. Games Appl., 3(4):523-536, 2013.

[17] A. Henrot. Extremum problems for eigenvalues of elliptic operators. Frontiers in Mathematics. Birkhäuser Verlag, Basel, 2006.

[18] M. Huang, P. E. Caines, and R. P. Malhamé. Large-population cost-coupled LQG problems with nonuniform agents: individual-mass behavior and decentralized $\epsilon$-Nash equilibria. IEEE Trans. Automat. Control, 52(9):1560-1571, 2007.

[19] M. Huang, R. P. Malhamé, and P. E. Caines. Large population stochastic dynamic games: closedloop McKean-Vlasov systems and the Nash certainty equivalence principle. Commun. Inf. Syst., 6(3):221-251, 2006.

[20] A. Lachapelle and M.-T. Wolfram. On a mean field game approach modeling congestion and aversion in pedestrian crowds. Transp. Res. Part B: Methodol., 45(10):1572 - 1589, 2011.

[21] J.-M. Lasry and P.-L. Lions. Jeux à champ moyen. I. Le cas stationnaire. C. R. Math. Acad. Sci. Paris, 343(9):619-625, 2006. 
[22] J.-M. Lasry and P.-L. Lions. Jeux à champ moyen. II. Horizon fini et contrôle optimal. C. R. Math. Acad. Sci. Paris, 343(10):679-684, 2006.

[23] J.-M. Lasry and P.-L. Lions. Mean field games. Jpn. J. Math., 2(1):229-260, 2007.

[24] P.-L. Lions. Cours au collège de france. http://www.college-de-france.fr.

[25] B. Noris, H. Tavares, S. Terracini, and G. Verzini. Uniform Hölder bounds for nonlinear Schrödinger systems with strong competition. Comm. Pure Appl. Math., 63(3):267-302, 2010.

[26] B. Noris, H. Tavares, S. Terracini, and G. Verzini. Convergence of minimax structures and continuation of critical points for singularly perturbed systems. J. Eur. Math. Soc. (JEMS), 14(4):1245-1273, 2012.

[27] B. Noris, H. Tavares, and G. Verzini. Existence and orbital stability of the ground states with prescribed mass for the $L^{2}$-critical and supercritical NLS on bounded domains. Anal. PDE, 7(8):18071838, 2014.

[28] B. Noris, H. Tavares, and G. Verzini. Stable solitary waves with prescribed $L^{2}$-mass for the cubic Schrödinger system with trapping potentials. Discrete Contin. Dyn. Syst., 35(12):6085-6112, 2015.

[29] V. Quitalo. A free boundary problem arising from segregation of populations with high competition. Arch. Ration. Mech. Anal., 210(3):857-908, 2013.

[30] P. H. Rabinowitz. Some global results for nonlinear eigenvalue problems. J. Functional Analysis, 7:487-513, 1971.

[31] N. Soave, H. Tavares, S. Terracini, and A. Zilio. Hölder bounds and regularity of emerging free boundaries for strongly competing Schrödinger equations with nontrivial grouping. arXiv:1506.00800, 062015.

[32] N. Soave and A. Zilio. Uniform bounds for strongly competing systems: the optimal Lipschitz case. Arch. Ration. Mech. Anal., 218(2):647-697, 2015.

[33] J. Wei and T. Weth. Asymptotic behaviour of solutions of planar elliptic systems with strong competition. Nonlinearity, 21(2):305-317, 2008.

marco.cirant@unimi.it

Dipartimento di Matematica, Università di Milano via Cesare Saldini 50, 20133 Milano (Italy)

gianmaria.verzini@polimi.it

Dipartimento di Matematica, Politecnico di Milano piazza Leonardo da Vinci 32, 20133 Milano (Italy) 